\title{
Expected topology of random real algebraic submanifolds
}

\author{
Damien Gayet, Jean-Yves Welschinger
}

November 10, 2018

\begin{abstract}
Let $X$ be a smooth complex projective manifold of dimension $n$ equipped with an ample line bundle $L$ and a rank $k$ holomorphic vector bundle $E$. We assume that $1 \leqslant k \leqslant n$, that $X, E$ and $L$ are defined over the reals and denote by $\mathbb{R} X$ the real locus of $X$. Then, we estimate from above and below the expected Betti numbers of the vanishing loci in $\mathbb{R} X$ of holomorphic real sections of $E \otimes L^{d}$, where $d$ is a large enough integer. Moreover, given any closed connected codimension $k$ submanifold $\Sigma$ of $\mathbb{R}^{n}$ with trivial normal bundle, we prove that a real section of $E \otimes L^{d}$ has a positive probability, independent of $d$, to contain around $\sqrt{d}^{n}$ connected components diffeomorphic to $\Sigma$ in its vanishing locus.
\end{abstract}

MAThematics SubJeCt ClAssification 2010: 14P25, 32Q15, 60 D05

KEYWORDS: Real projective manifold, ample line bundle, random polynomial, Betti numbers

\section{Contents}

1 Introduction 2

1.1 Upper estimates . . . . . . . . . . . . . . . . . 3

1.2 Lower estimates and topology . . . . . . . . . . . . . . . . 3

1.3 Some related results ......................... 4

2 Lower estimates for the expected Betti numbers 5

2.1 Statement of the results . . . . . . . . . . . . 5

2.1.1 Framework .................... 5

2.1 .2 The lower estimates . . . . . . . . . . . . . . . . . . . . . . . 6

2.2 Closed affine real algebraic submanifolds . . . . . . . . . . . . 6

2.3 Hörmander sections . . . . . . . . . . . . . . . . . . . . . . . . . . . . . . . . .

2.4 Proof of Theorem $1.2 .1 \ldots \ldots \ldots \ldots \ldots$

2.5 Proof of Theorem 2.1.1 . . . . . . . . . . . . . . . . . . . 14

2.6 Proof of Corollary $1.2 .2 \ldots \ldots \ldots \ldots \ldots$

3 Upper estimates for the expected Betti numbers

3.1 Statement of the results . . . . . . . . . . . . 17

3.2 Incidence varieties . . . . . . . . . . . . . . . . . . . . . . . . 19 
3.3 Computation of the Jacobian determinants . . . . . . . . . . . 21

3.3.1 Jacobian determinant of $\rho_{x} \ldots \ldots \ldots . \ldots . \ldots 21$

3.3.2 Jacobian determinant of the evaluation map . . . . . . . . . 23

3.4 Proof of Theorem $3.1 .3 \ldots \ldots \ldots \ldots$

3.4 .1 The case $k<n \ldots \ldots \ldots \ldots \ldots$

3.4 .2 The case $k=n \ldots \ldots \ldots \ldots$. . . . . . . . . . . . . . . .

3.5 Equidistribution of critical points in the complex case . . . . . . 26

\section{Introduction}

Let $X$ be a smooth complex projective manifold of positive dimension $n$ equipped with an ample line bundle $L$ and let $E$ be a holomorphic vector bundle of rank $k$ over $X$. From the vanishing theorem of Kodaira and Serre, we know that the dimension $N_{d}$ of the complex vector space $H^{0}\left(X, E \otimes L^{d}\right)$ of global holomorphic sections of $E \otimes L^{d}$ grows as a polynomial of degree $n$ in $d$. We will assume throughout this paper that $1 \leqslant k \leqslant n$ and that $X, E$ and $L$ are defined over the reals. We denote by $\mathbb{R} X$ the real locus of $X$ and by $\mathbb{R} H^{0}\left(X, E \otimes L^{d}\right)$ the real vector space of real holomorphic sections of $E \otimes L^{d}$, see (5). Its dimension equals $N_{d}$. The discriminant locus $\mathbb{R} \Delta_{d} \subset \mathbb{R} H^{0}\left(X, E \otimes L^{d}\right)$ of sections which do not vanish transversally is a codimension one submanifold for $d$ large enough and for every $\sigma$ in its complement, the real vanishing locus $\mathbb{R} C_{\sigma}$ of $\sigma$ is a smooth codimension $k$ submanifold of $\mathbb{R} X$. The topology of $\mathbb{R} C_{\sigma}$ drastically depends on the choice of $\sigma \in \mathbb{R} H^{0}\left(X, E \otimes L^{d}\right) \backslash \mathbb{R} \Delta_{d}$. When $n=k=1, X=\mathbb{C} P^{1}, L=\mathcal{O}_{\mathbb{C} P^{1}}(1)$ and $E=\mathcal{O}_{\mathbb{C} P^{1}}$ for example, $\sigma$ is a real polynomial of degree $d$ in one variable and $\mathbb{R} C_{\sigma}$ the set of its real roots.

The space $\mathbb{R} H^{0}\left(X, E \otimes L^{d}\right)$ inherits classical probability measures. Indeed, let $h_{E}$ be a Hermitian metric on $E$ and $h_{L}$ be a Hermitian metric of positive curvature on $L$, both $h_{E}$ and $h_{L}$ being real, that is invariant under the $\mathbb{Z} / 2 \mathbb{Z}$-Galois action of $E$ and $L$. We denote by $h_{E, d}=h_{E} \otimes h_{L}^{d}$ the induced metric on $E \otimes L^{d}$. Then, the vector space $\mathbb{R} H^{0}\left(X, E \otimes L^{d}\right)$ becomes Euclidean, with the $L^{2}$-scalar product defined by

$$
\forall \sigma, \tau \in \mathbb{R} H^{0}\left(X, E \otimes L^{d}\right),\langle\sigma, \tau\rangle=\int_{X} h_{E, d}(\sigma, \tau) d x
$$

where $d x$ denotes any chosen volume form on $X$ (our results being asymptotic in $d$, they turn out not to depend on the choice of $d x)$. It thus inherits a Gaussian probability measure $\mu_{\mathbb{R}}$ whose density at $\sigma \in \mathbb{R} H^{0}\left(X, E \otimes L^{d}\right)$ with respect to the Lebesgue measure is $\frac{1}{\sqrt{\pi}^{N_{d}}} e^{-\|\sigma\|^{2}}$.

What is the typical topology of $\mathbb{R} C_{\sigma}$ for $\sigma \in \mathbb{R} H^{0}\left(X, E \otimes L^{d}\right)$ chosen at random for $d \mu_{\mathbb{R}}$ ? We do not know, but can estimate its average Betti numbers. To formulate our results, let us denote, for every $i \in\{0, \cdots, n-k\}$, by $b_{i}\left(\mathbb{R} C_{\sigma}, \mathbb{R}\right)=\operatorname{dim} H_{i}\left(\mathbb{R} C_{\sigma}, \mathbb{R}\right)$ the $i$-th Betti number of $\mathbb{R} C_{\sigma}$ and by

$$
\mathbb{E}\left(b_{i}\right)=\int_{\mathbb{R} H^{0}\left(X, E \otimes L^{d}\right) \backslash \mathbb{R} \Delta_{d}} b_{i}\left(\mathbb{R} C_{\sigma}, \mathbb{R}\right) d \mu_{\mathbb{R}}(\sigma)
$$

its expected value. 


\section{$1.1 \quad$ Upper estimates}

As in [14], for every $i \in\{0, \cdots, n-k\}$, we denote by $\operatorname{Sym}_{\mathbb{R}}(i, n-k-i)$ the open cone of real symmetric matrices of size $n-k$ and signature $(i, n-k-i)$, by $\mu_{\mathbb{R}}$ the classical Gaussian measure on the space of real symmetric matrices and by $e_{\mathbb{R}}(i, n-k-i)$ the numbers

$$
e_{\mathbb{R}}(i, n-k-i)=\int_{S m_{\mathbb{R}}(i, n-k-i)}|\operatorname{det} A| d \mu_{\mathbb{R}}(A),
$$

see 93.1 . We then denote by $\operatorname{Vol}_{h_{L}}(\mathbb{R} X)$ the volume of $\mathbb{R} X$ for the Riemannian metric induced by the Kähler metric $g_{h_{L}}$ defined by the curvature form of $h_{L}$, see (3) and (4).

Theorem 1.1.1 Let $X$ be a smooth real projective manifold of dimension $n,\left(L, h_{L}\right)$ be a real holomorphic Hermitian line bundle of positive curvature over $X$ and $\left(E, h_{E}\right)$ be a rank $k$ real holomorphic Hermitian vector bundle, with $1 \leqslant k \leqslant n, k \neq n$. Then, for every $0 \leqslant i \leqslant n-k$,

$$
\limsup _{d \rightarrow \infty} \frac{1}{\sqrt{d}^{n}} \mathbb{E}\left(b_{i}\right) \leqslant\left(\begin{array}{l}
n-1 \\
k-1
\end{array}\right) e_{\mathbb{R}}(i, n-k-i) \frac{V o l_{h_{L}}(\mathbb{R} X)}{V o l_{F S}\left(\mathbb{R} P^{k}\right)} .
$$

Moreover, when $k=n, \frac{1}{\sqrt{d}^{n}} \mathbb{E}\left(b_{0}\right)$ converges to $\frac{V_{l_{h_{L}}}(\mathbb{R} X)}{\operatorname{Vol}_{F S}\left(\mathbb{R} P^{n}\right)}$ as d grows to infinity.

In fact, the right hand side of the inequality given by Theorem 1.1.1 also involves the determinant of random matrices of size $k-1$ and the volume of the Grassmann manifold of $(k-1)$ linear subspaces of $\mathbb{R}^{n-1}$, see Theorem 3.1 .2 , but these can be computed explicitly. Note that when $E$ is the trivial line bundle, Theorem 1.1.1 reduces to Theorem 1.1 of [14].

Theorem 1.1.1 relies on Theorem 3.1.3, which establishes the asymptotic equidistribution of clouds of critical points, see \$3.1. We obtain a similar result in a complex projective setting, for critical points of Lefschetz pencils, see Theorem 3.5.1.

\subsection{Lower estimates and topology}

Let $\Sigma$ be a closed submanifold of codimension $k$ of $\mathbb{R}^{n}, 1 \leqslant k \leqslant n$, which we do not assume to be connected. For every $\sigma \in \mathbb{R} H^{0}\left(X, E \otimes L^{d}\right) \backslash \mathbb{R} \Delta_{d}$, we denote by $N_{\Sigma}(\sigma)$ the maximal number of disjoint open subsets of $\mathbb{R} X$ having the property that each such open subset $U^{\prime}$ contains a codimension $k$ submanifold $\Sigma^{\prime}$ such that $\Sigma^{\prime} \subset \mathbb{R} C_{\sigma}$ and $\left(U^{\prime}, \Sigma^{\prime}\right)$ is diffeomorphic to $\left(\mathbb{R}^{n}, \Sigma\right)$. We then set

$$
\mathbb{E}\left(N_{\Sigma}\right)=\int_{\mathbb{R} H^{0}\left(X, E \otimes L^{d}\right) \backslash \mathbb{R} \Delta_{d}} N_{\Sigma}(\sigma) d \mu_{\mathbb{R}}(\sigma)
$$

and we associate to $\Sigma$, in fact to its isotopy class in $\mathbb{R}^{n}$, a constant $c_{\Sigma}$ which is positive if and only if $\Sigma$ has trivial normal bundle in $\mathbb{R}^{n}$, see (14) for its definition and Lemma 2.2.3. The latter measures à la Donaldson the amount of transversality that a polynomial map $\mathbb{R}^{n} \rightarrow \mathbb{R}^{k}$ vanishing along a submanifold isotopic to $\Sigma$ may have. 
Theorem 1.2.1 Let $X$ be a smooth real projective manifold of dimension $n,\left(L, h_{L}\right)$ be a real holomorphic Hermitian line bundle of positive curvature over $X$ and $\left(E, h_{E}\right)$ be a rank $k$ real holomorphic Hermitian vector bundle, with $1 \leqslant k \leqslant n$. Let $\Sigma$ be a closed submanifold of codimension $k$ of $\mathbb{R}^{n}$ with trivial normal bundle, which does not need to be connected. Then,

$$
\liminf _{d \rightarrow \infty} \frac{1}{\sqrt{d}^{n}} \mathbb{E}\left(N_{\Sigma}\right) \geqslant c_{\Sigma} V o l_{h_{L}}(\mathbb{R} X) .
$$

In particular, when $\Sigma$ is connected, Theorem 1.2.1 bounds from below the expected number of connected components diffeomorphic to $\Sigma$ in the real vanishing locus of a random section $\sigma \in \mathbb{R} H^{0}\left(X, E \otimes L^{d}\right)$. The constant $c_{\Sigma}$ does not depend on the choice of the triple $\left(X,\left(L, h_{L}\right),\left(E, h_{E}\right)\right)$, it only depends on $\Sigma$. When $k=1$ and $E=\mathcal{O}_{X}$, Theorem 1.2.1 coincides with Theorem 1.2 of [16]. Computing $c_{\Sigma}$ for explicit submanifolds $\Sigma$ yields the following lower bounds for the Betti numbers.

Corollary 1.2.2 Under the hypotheses of Theorem 1.2.1, for every $i \in\{0, \cdots, n-k\}$,

$$
\liminf _{d \rightarrow \infty} \frac{1}{\sqrt{d}^{n}} \mathbb{E}\left(b_{i}\right) \geqslant \exp \left(-e^{84+6 n}\right) \operatorname{Vol}_{h_{L}}(\mathbb{R} X) .
$$

\subsection{Some related results}

The case $X=\mathbb{C} P^{1}, E=\mathcal{O}_{\mathbb{C} P^{1}}$ and $L=\mathcal{O}_{\mathbb{C} P^{1}}(1)$ was first considered by M. Kac in [18] for a different measure. In this case and with our measure, Kostlan [19] and Shub and Smale [34] gave an exact formula for the mean number of real roots of a polynomial, as well as the mean number of intersection points of $n$ hypersurfaces in $\mathbb{R} P^{n}$. Still in $\mathbb{R} P^{n}$, Podkorytov 27] computed the mean Euler characteristics of random algebraic hypersufaces, and Bürgisser [4] extented this result to complete intersections. In [13], we proved the exponential rarefaction of real curves with a maximal number of components in real algebraic surfaces. In [15] and [14], we bounded from above the mean Betti numbers of random real hypersurfaces in real projective manifolds and in [16], we gave a lower bound for them.

A similar probabilistic study of complex projective manifolds has been performed by Shiffman and Zelditch, see [30, [33, [2] for example, or also [3], 36]. In particular, the asymptotic equidistribution of critical points of random sections over a fixed projective manifold has been studied in [8], [9] and [22], or also [11, [1], [5], while we studied critical points of the restriction of a fixed Morse function on random real hypersurfaces, see [15], [14].

A similar question concerns the mean number of components of the vanishing locus of eigenfunctions of the Laplacian. It has been studied on the round sphere by Nazarov and Sodin [25] (see also [35]), Lerario and Lundberg [20] or Sarnak and Wigman [28]. In a general Riemannian setting, Zelditch proved in [38] the equidistribution of the vanishing locus, whereas critical points of random eigenfunctions of the Laplacian have been addressed by Nicolaescu in [26].

Section 2 is devoted to lower estimates and the proof of Theorem 1.2.1. In this proof, the $L^{2}$-estimates of Hörmander play a crucial rôle, see $\$ 2.3$, and we follow the same approach as in [16] (see also [12] for a similar construction). Section 3 is 
devoted to upper estimates and the proof of Theorem 1.1.1.

Aknowledgements. The research leading to these results has received funding from the European Community's Seventh Framework Progamme ([FP7/2007-2013] [FP7/2007-2011]) under grant agreement $n^{\circ}$ [258204].

\section{Lower estimates for the expected Betti numbers}

\subsection{Statement of the results}

\subsubsection{Framework}

Let us first recall our framework. We denote by $X$ a smooth complex projective manifold of dimension $n$ defined over the reals, by $c_{X}: X \rightarrow X$ the induced Galois antiholomorphic involution and by $\mathbb{R} X=\operatorname{Fix}\left(c_{X}\right)$ the real locus of $X$ which we implicitly assume to be non-empty. We then consider an ample line bundle $L$ over $X$, also defined over the reals. It comes thus equipped with an antiholomorphic involution $c_{L}: L \rightarrow L$ which turns the bundle projection map $\pi: L \rightarrow X$ into a $\mathbb{Z} / 2 \mathbb{Z}$-equivariant one, so that $c_{X} \circ \pi=\pi \circ c_{L}$. We equip $L$ in addition with a real Hermitian metric $h_{L}$, thus invariant under $c_{L}$, which has a positive curvature form $\omega$ locally defined by

$$
\omega=\frac{1}{2 i \pi} \partial \bar{\partial} \log h_{L}(e, e)
$$

for any non-vanishing local holomorphic section $e$ of $L$. This metric induces a Kähler metric

$$
g_{h_{L}}=\omega(., i .)
$$

on $X$, which reduces to a Riemannian metric $g_{h_{L}}$ on $\mathbb{R} X$. Let finally $E$ be a holomorphic vector bundle of rank $k, 1 \leqslant k \leqslant n$, defined over the reals and equipped with a antiholomorphic involution $c_{E}$ and a real Hermitian metric $h_{E}$. For every $d>0$, we denote by

$$
\mathbb{R} H^{0}\left(X, E \otimes L^{d}\right)=\left\{\sigma \in H^{0}\left(X, E \otimes L^{d}\right) \mid\left(c_{E} \otimes c_{L^{d}}\right) \circ \sigma=\sigma \circ c_{X}\right\}
$$

the space of global real holomorphic sections of $E \otimes L^{d}$. It is equipped with the $L^{2}$-scalar product defined by the formula

$$
\forall(\sigma, \tau) \in \mathbb{R} H^{0}\left(X, E \otimes L^{d}\right),\langle\sigma, \tau\rangle=\int_{X} h_{E, d}(\sigma, \tau)(x) d x,
$$

where $h_{E, d}=h_{E} \otimes h_{L}^{d}$. Here, $d x$ denotes any volume form of $X$. For instance, $d x$ can be chosen to be the normalized volume form $d V_{h_{L}}=\frac{\omega^{n}}{\int_{X} \omega^{n}}$. This $L^{2}$-scalar product finally induces a Gaussian probability measure $\mu_{\mathbb{R}}$ on $\mathbb{R} H^{0}\left(X, E \otimes L^{d}\right)$ whose density with respect to the Lebesgue one at $\sigma \in \mathbb{R} H^{0}\left(X, E \otimes L^{d}\right)$ writes $\frac{1}{\sqrt{\pi^{N}}} e^{-\|\sigma\|^{2}}$, where $N_{d}=\operatorname{dim} H^{0}\left(X, E \otimes L^{d}\right)$. It is with respect to this probability measure that we consider random real codimension $k$ submanifolds (as in the works [19] and [34, [15], [14] and [16]). 


\subsubsection{The lower estimates}

The aim of Section 2 is to prove Theorem 1.2.1. In addition to Theorem 1.2.1, we also get the following Theorem 2.1.1, which is a consequence of Proposition 2.4.2 below.

Theorem 2.1.1 Under the hypotheses of Theorem 1.2.1, for every $0 \leqslant \epsilon<1$,

$$
\liminf _{d \rightarrow \infty} \mu_{\mathbb{R}}\left\{\sigma \in \mathbb{R} H^{0}\left(X, E \otimes L^{d}\right) \mid N_{\Sigma}(\sigma) \geqslant \epsilon c_{\Sigma} V o l_{h_{L}}(\mathbb{R} X) \sqrt{d}^{n}\right\}>0
$$

In fact, the positive lower bound given by Theorem 2.1.1 can be made explicit, see (30).

Let us now denote, for every $1 \leqslant k \leqslant n$, by $\mathcal{H}_{n, k}$ the set of diffeormophism classes of smooth closed connected codimension $k$ submanifolds of $\mathbb{R}^{n}$. For every $i \in\{0, \cdots, n-k\}$ and every $[\Sigma] \in \mathcal{H}_{n, k}$, we denote by $b_{i}(\Sigma)=\operatorname{dim} H_{i}(\Sigma ; \mathbb{R})$ its $i$-th Betti number with real coefficients and by $m_{i}(\Sigma)$ its $i$-th Morse number. This is the infimum over all Morse functions $f$ on $\Sigma$ of the number of critical points of index $i$ of $f$. Then, we set $c_{[\Sigma]}=\sup _{\Sigma \in[\Sigma]} c_{\Sigma}$ and

$$
\mathbb{E}\left(m_{i}\right)=\int_{\mathbb{R} H^{0}\left(X, E \otimes L^{d}\right) \backslash \mathbb{R} \Delta_{d}} m_{i}\left(\mathbb{R} C_{\sigma}\right) d \mu_{\mathbb{R}}(\sigma) .
$$

Corollary 2.1.2 Let $X$ be a smooth real projective manifold of dimension $n,\left(L, h_{L}\right)$ be a real holomorphic Hermitian line bundle of positive curvature over $X$ and $\left(E, h_{E}\right)$ be a rank $k$ real holomorphic Hermitian vector bundle, with $1 \leqslant k \leqslant n$. Then, for every $i \in\{0, \cdots, n-k\}$,

$$
\begin{aligned}
\liminf _{d \rightarrow \infty} \frac{1}{\sqrt{d}^{n}} \mathbb{E}\left(b_{i}\right) & \geqslant\left(\sum_{[\Sigma] \in \mathcal{H}_{n, k}} c_{[\Sigma]} b_{i}(\Sigma)\right) V o l_{h_{L}}(\mathbb{R} X) \text { and likewise } \\
\liminf _{d \rightarrow \infty} \frac{1}{\sqrt{d}^{n}} \mathbb{E}\left(m_{i}\right) & \geqslant\left(\sum_{[\Sigma] \in \mathcal{H}_{n, k}} c_{[\Sigma]} m_{i}(\Sigma)\right) V o l_{h_{L}}(\mathbb{R} X) .
\end{aligned}
$$

Note that in Corollary 2.1.2, we could have chosen one representative $\Sigma$ in each diffeomorphism class $[\Sigma] \in \mathcal{H}_{n, k}$ and obtained the lower estimates (77), (8) with constants $c_{\Sigma}$ instead of $c_{[\Sigma]}$. But it turns out that in the proof of Corollary 2.1.2 we are free to choose the representative we wish in every diffeomorphism class and that the higher $c_{\Sigma}$ is, the better the estimates (7), (8) are. This is why we introduce the constant $c_{[\Sigma]}$, which is positive if and only if $[\Sigma]$ has a representative $\Sigma$ with trivial normal bundle in $\mathbb{R}^{n}$, see (14) and Lemma 2.2.3.

\subsection{Closed affine real algebraic submanifolds}

We introduce here the notion of regular pair, see Definition 2.2.1, and the constant $c_{\Sigma}$ associated to any isotopy class of smooth closed codimension $k$ submanifold $\Sigma$ of $\mathbb{R}^{n}$, see (14).

Definition 2.2.1 Let $U$ be a bounded open subset of $\mathbb{R}^{n}$ and $P \in \mathbb{R}\left[x_{1}, \cdots x_{n}\right]^{k}$, $1 \leqslant k \leqslant n$. The pair $(U, P)$ is said to be regular if and only if 
1. zero is a regular value of the restriction of $P$ to $U$,

2. the vanishing locus of $P$ in $U$ is compact.

Hence, for every regular pair $(U, P)$, the vanishing locus of $P$ does not intersect the boundary of $U$ and it meets $U$ in a smooth compact codimension $k$ submanifold.

In the sequel, for every integer $p$ and every vector $v \in \mathbb{R}^{p}$, we denote by $|v|$ its Euclidian norm, and for every integers $p$ and $q$, and every linear map $F: \mathbb{R}^{p} \rightarrow \mathbb{R}^{q}$, we denote by $F^{*}$ the adjoint of $F$, defined by the property

$$
\forall v \in \mathbb{R}^{p}, \forall w \in \mathbb{R}^{q},\langle F(v), w\rangle=\left\langle v, F^{*}(w)\right\rangle,
$$

and denote by $\|F\|$ its operator norm, that is

$$
\|F\|=\sup _{v \in \mathbb{R}^{p} \backslash\{0\}}|F(v)| /|v| .
$$

We will also use the norm

$$
\|F\|_{2}=\sqrt{\operatorname{Tr} F F^{*}} .
$$

These norms satisfy $\|F\| \leqslant\|F\|_{2}$. Finally, if $P=\left(P_{1}, \cdots, P_{k}\right) \in \mathbb{R}\left[x_{1}, \cdots, x_{n}\right]^{k}$, we denote by $\|P\|_{L^{2}}$ its $L^{2}$-norm defined by

$$
\|P\|_{L^{2}}^{2}=\int_{\mathbb{C}^{n}}|P(z)|^{2} e^{-\pi|z|^{2}} d z=\sum_{i=1}^{k} \int_{\mathbb{C}^{n}}\left|P_{i}(z)\right|^{2} e^{-\pi|z|^{2}} d z=\sum_{i=1}^{k}\left\|P_{i}\right\|_{L^{2}}^{2} .
$$

Definition 2.2.2 For every regular pair $(U, P)$ given by Definition 2.2.1, we denote by $\mathcal{T}_{(U, P)}$ the set of $(\delta, \epsilon) \in\left(\mathbb{R}_{+}^{*}\right)^{2}$ such that

1. there exists a compact subset $K$ of $U$ satisfying $\inf _{x \in U \backslash K}|P(x)|>\delta$,

2. for every $y \in U,|P(y)|<\delta \Rightarrow \forall w \in \mathbb{R}^{k},\left|\left(d_{\mid y} P\right)^{*}(w)\right| \geqslant \epsilon|w|$.

Hence, for every regular pair $(U, P)$ given by Definition $2.2 .1,(\delta, \epsilon)$ belongs to $\mathcal{T}_{(U, P)}$ provided the $\delta$-sublevel of $P$ does not intersect the boundary of $U$ while inside this $\delta$-sublevel, $P$ is in a sense $\epsilon$-far from having a critical point. This quantifies how much transversally $P$ vanishes in a way similar to the one used by Donaldson in [7].

Then, for every regular pair $(U, P)$, we set $R_{(U, P)}=\max \left(1, \sup _{y \in U}|y|\right)$, so that $U$ is contained in the ball centered at the origin and of radius $R_{(U, P)}$. Finally, we set

$$
\tau_{(U, P)}=24 k \rho_{R_{(U, P)}}\|P\|_{L^{2}}^{2} \inf _{(\delta, \epsilon) \in \mathcal{T}_{(U, P)}}\left(\frac{1}{\delta^{2}}+\frac{\pi n}{\epsilon^{2}}\right) \in \mathbb{R}_{+}^{*},
$$

where, for every $R>0$,

$$
\begin{gathered}
\rho_{R}=\inf _{\mathbb{R}^{+}} g_{R}, \\
g_{R}: s \in \mathbb{R}_{+}^{*} \mapsto \frac{(R+s)^{2 n}}{s^{2 n}} e^{\pi(R+s)^{2}},
\end{gathered}
$$

so that

$$
e^{\pi R^{2}} \leqslant \rho_{R} \leqslant 4^{n} e^{4 \pi R^{2}}
$$


This constant $\tau_{(U, P)}$ is the main ingredient in the definition of $c_{\Sigma}$, see (14). The lower $\tau_{(U, P)}$ is, the larger $c_{\Sigma}$ is and the better the estimates given by Theorem 1.2.1 are. Note that $\tau_{(U, P)}$ remains small whenever $\delta, \epsilon$ are not too small, that is when $P$ vanishes quite transversally in $U$.

Now, let $\Sigma$ be a closed submanifold of codimension $k$ of $\mathbb{R}^{n}$, not necessarily connected. We denote by $\mathcal{I}_{\Sigma}$ the set of regular pairs $(U, P)$ given by Definition 2.2.1, such that the vanishing locus of $P$ in $U$ contains a subset isotopic to $\Sigma$ in $\mathbb{R}^{n}$.

Lemma 2.2.3 Let $\Sigma$ be a closed submanifold of codimension $k>0$ of $\mathbb{R}^{n}$, not necessarily connected. Then, $\mathcal{I}_{\Sigma}$ is non empty if and only if the normal bundle of $\Sigma$ in $\mathbb{R}^{n}$ is trivial.

Proof. If $(U, P) \in \mathcal{I}_{\Sigma}$, then $P: \mathbb{R}^{n} \rightarrow \mathbb{R}^{k}$ contains in its vanishing locus a codimension $k$ submanifold $\hat{\Sigma}$ which is isotopic to $\Sigma$ in $\mathbb{R}^{n}$. The normal bundle of $\Sigma$ in $\mathbb{R}^{n}$ is thus trivial if and only if the normal bundle of $\widehat{\Sigma}$ in $\mathbb{R}^{n}$ is trivial. But the differential of $P$ at every point of $\widehat{\Sigma}$ provides an isomorphism between the normal bundle of $\widehat{\Sigma}$ in $\mathbb{R}^{n}$ and the product $\hat{\Sigma} \times \mathbb{R}^{k}$.

Conversely, if $\Sigma$ has a trivial normal bundle in $\mathbb{R}^{n}$, it has been proved by Seifert [29] (see also [24]) that there exist a polynomial map $P: \mathbb{R}^{n} \rightarrow \mathbb{R}^{k}$ and a tubular neighbourhood $U$ of $\Sigma$ in $\mathbb{R}^{n}$ such that $P^{-1}(0) \cap U$ is isotopic to $\Sigma$ in $U$. The strategy of the proof is to first find a smooth function $U \rightarrow \mathbb{R}^{k}$ in a neighborhood of $\Sigma$ which vanishes transversally along $\Sigma$ and then to suitably approximate the coordinates of this function by some polynomial, see [29], 24]. The pair $(U, P)$ then belongs to $\mathcal{I}_{\Sigma}$ by Definition 2.2.1.

We then set $c_{\Sigma}=0$ if $\Sigma$ does not have a trivial normal bundle in $\mathbb{R}^{n}$ and

$$
c_{\Sigma}=\sup _{(U, P) \in \mathcal{I}_{\Sigma}}\left(\frac{m_{\tau_{(U, P)}}}{2^{n} \operatorname{Vol}\left(B\left(R_{(U, P)}\right)\right)}\right) \text { otherwise, }
$$

where $\operatorname{Vol}\left(B\left(R_{(U, P)}\right)\right)$ denotes the volume of the Euclidean ball of radius $R_{(U, P)}$ in $\mathbb{R}^{n}$, and where, for every $\tau>0$,

$$
m_{\tau}=\sup _{[\sqrt{\tau},+\infty[} f_{\tau}
$$

with $f_{\tau}: a \in\left[\sqrt{\tau},+\infty\left[\mapsto \frac{1}{\sqrt{\pi}}\left(1-\frac{\tau}{a^{2}}\right) \int_{a}^{+\infty} e^{-t^{2}} d t\right.\right.$. For large values of $m_{\tau}$, as the ones which appear in 2.6 , the estimate

$$
c_{\Sigma} \geqslant e^{-2 \tau_{(U, P)}}
$$

holds, compare (2.8) of [16].

\subsection{Hörmander sections}

Our key tool to prove Theorems 1.1.1 and 1.2.1 has been developped by L. Hörmander. We introduce in this 2.3 the material we need. For every positive $d$ and every $\sigma \in \mathbb{R} H^{0}\left(X, E \otimes L^{d}\right)$, we set

$$
\|\sigma\|_{L^{2}\left(h_{L}\right)}^{2}=\int_{X}\|\sigma\|_{h_{E, d}}^{2} d V_{h_{L}}
$$


where $d V_{h_{L}}=\omega^{n} / \int_{X} \omega^{n}$, compare (6). Let us choose a field of $h_{L}$-trivializations of $L$ on $\mathbb{R} X$ given by Definition 4 of [16]. It provides in particular, for every $x \in \mathbb{R} X$, a local holomorphic chart $\psi_{x}:\left(W_{x}, x\right) \subset X \rightarrow\left(V_{x}, 0\right) \subset \mathbb{C}^{n}$ isometric at $x$, and a non-vanishing holomorphic section $e$ of $L$ defined over $W_{x}$ such that $\phi=-\log h_{L}(e, e)$ vanishes at $x$ and is positive elsewhere. Moreover, there exist a positive constant $\alpha_{1}$ such that

$$
\forall y \in V_{x},\left.\left.\left|\phi \circ \psi_{x}^{-1}(y)-\pi\right| y\right|^{2}\left|\leqslant \alpha_{1}\right| y\right|^{3} .
$$

Restricting $W_{x}$ if necessary, we choose a holomophic trivialization $\left(e_{1}, \cdots, e_{k}\right)$ of $E_{\mid W_{x}}$ which is orthonormal at $x$. This provides a trivialization $\left(e_{1} \otimes e^{d}, \cdots, e_{k} \otimes e^{d}\right)$ of $E \otimes L_{\mid W_{x}}^{d}$. In this trivialization, the restriction of $\sigma$ to $W_{x}$ writes

$$
\sigma=\sum_{j=1}^{k} f_{\sigma}^{j} e_{j} \otimes e^{d}
$$

for some holomorphic functions $f_{\sigma}^{j}: W_{x} \rightarrow \mathbb{C}$, We write $f_{\sigma}=\left(f_{\sigma}^{1}, \cdots, f_{\sigma}^{k}\right)$ and we set

$$
|\sigma|=\left|f_{\sigma}\right|
$$

so that on $W_{x},\|\sigma\|_{h_{E, d}}^{2}=\left\|\sum_{j=1}^{k} f_{\sigma}^{k} e_{j}\right\|_{h_{E}}^{2} e^{-d \phi}$ and $\|\sigma(x)\|_{h_{E, d}}^{2}=|\sigma(x)|^{2}$ since the frames $\left(e_{1}, \cdots, e_{k}\right)$ and $e$ are orthonormal at the point $x$ so that in particular $\phi(x)=0$. For every $z \in W_{x}$, we define

$$
\begin{aligned}
\left\|d_{\mid z} \sigma\right\|_{2} & =\left\|d_{\mid y}\left(f_{\sigma} \circ \psi_{x}^{-1}\right)\right\|_{2}, \\
\left\|d_{\mid z} \sigma\right\| & =\left\|d_{\mid y}\left(f_{\sigma} \circ \psi_{x}^{-1}\right)\right\|,
\end{aligned}
$$

and

$$
\left(d_{\mid z} \sigma\right)^{*}=\left(d_{\mid y}\left(f_{\sigma} \circ \psi_{x}^{-1}\right)\right)^{*},
$$

where $y=\psi_{x}(z)$. Finally, we denote, for every small enough $r>0$, by $B(x, r) \subset W_{x}$ the ball centered at $x$ and of radius $r$ for the flat metric of $V_{x}$ pulled back by $\psi_{x}$, so that

$$
B(x, r)=\psi_{x}^{-1}(B(0, r)) .
$$

Proposition 2.3.1 Let $X$ be a smooth real projective manifold of dimension $n,\left(L, h_{L}\right)$ be a real holomorphic Hermitian line bundle of positive curvature over $X$ and $\left(E, h_{E}\right)$ be a rank $k$ real holomorphic Hermitian vector bundle, with $1 \leqslant k \leqslant n$. We choose a field of $h_{L}$-trivializations on $\mathbb{R} X$. Then, for every regular pair $(U, P)$, every large enough integer $d$, every $x$ in $\mathbb{R} X$ and every local trivialization of $E$ orthonormal at $x$, there exist $\sigma_{(U, P)} \in \mathbb{R} H^{0}\left(X, E \otimes L^{d}\right)$ and an open subset $U_{d}$ of $B\left(x, \frac{R_{(U, P)}}{\sqrt{d}}\right) \cap \mathbb{R} X$ such that

1. $\left\|\sigma_{(U, P)}\right\|_{L^{2}\left(h_{L}\right)}$ be equivalent to $\frac{\|P\|_{L^{2}}}{\sqrt{\delta_{L}}}$ as d grows to infinity, where $\|P\|_{L^{2}}$ is defined by (9) and $\delta_{L}=\int_{X} \omega^{n}$,

2. $\left(U_{d}, \sigma_{(U, P)}^{-1}(0) \cap U_{d}\right)$ be diffeomorphic to $\left(U, P^{-1}(0) \cap U\right) \subset \mathbb{R}^{n}$, 
3. for every $(\delta, \epsilon) \in \mathcal{T}_{(U, P)}$ given by Definition 2.2.2, there exists a compact subset $K_{d} \subset U_{d}$ such that

$$
\inf _{U_{d} \backslash K_{d}}\left|\sigma_{(U, P)}\right|>\frac{\delta}{2} \sqrt{d}^{n}
$$

while for every $y$ in $U_{d}$,

$$
\left|\sigma_{(U, P)}(y)\right|<\frac{\delta}{2} \sqrt{d}^{n} \Rightarrow \forall w \in \mathbb{R}^{k},\left|\left(d_{\mid y} \sigma_{(U, P)}\right)^{*}(w)\right| \geqslant \frac{\epsilon}{2} \sqrt{d}^{n+1}|w| .
$$

Proof. We proceed as in the proof of Proposition 3.2 of [16]. Let $(U, P)$ be a regular pair, $x \in \mathbb{R} X$ and $d$ large enough. We set $U_{d}=\psi_{x}^{-1}\left(\frac{1}{\sqrt{d}} U\right) \subset B\left(x, \frac{R_{(U, P)}}{\sqrt{d}}\right)$ and $K_{d}=\psi_{x}^{-1}\left(\frac{1}{\sqrt{d}} K\right)$. Let $\chi: \mathbb{C}^{n} \rightarrow[0,1]$ be a smooth function with compact support in $B\left(0, R_{(U, P)}\right)$, which equals one in a neighbourhood of the origin. Then, let $\sigma$ be the global smooth section of $E \otimes L^{d}$ defined by $\sigma_{\mid X \backslash W_{x}}=0$ and

$$
\sigma_{\mid W_{x}}=\left(\chi \circ \psi_{x}\right)\left(\sum_{j=1}^{k} P_{j}\left(\sqrt{d} \psi_{x}\right) e_{j} \otimes e^{d}\right),
$$

where $P=\left(P_{1}, \cdots, P_{k}\right)$ is now considered as a function $\mathbb{C}^{n} \rightarrow \mathbb{C}^{k}$. From the $L^{2}$ estimates of Hörmander, see [17] or [21, there exists a global section $\tau$ of $E \otimes L^{d}$ such that $\bar{\partial} \tau=\bar{\partial} \sigma$ and $\|\tau\|_{L^{2}\left(h_{E, d}\right)} \leqslant\|\bar{\partial} \sigma\|_{L^{2}\left(h_{E, d}\right)}$ for $d$ large enough. This section $\tau$ can be chosen orthogonal to holomorphic sections and is then unique, in particular real. Moreover, there exist positive constants $c_{1}$ and $c_{2}$, which do not depend on $x$, such that $\|\tau\|_{L^{2}\left(h_{E, d}\right)} \leqslant c_{1} e^{-c_{2} d}$ and $\sup _{U_{d}}\left(|\tau|+\|\tau\|_{2}\right) \leqslant c_{2} e^{-c_{2} d}$, see Lemma 3.3 of [16]. We then set $\sigma_{(U, P)}=\sqrt{d}^{n}(\sigma-\tau)$. It has the desired properties as can be checked along the same lines as in the proof of Proposition 3.2 of [16] and thanks to Lemma 2.3.2.

Lemma 2.3.2 Let $U$ be an open subset of $\mathbb{R}^{n}, 1 \leqslant k \leqslant n, f: U \rightarrow \mathbb{R}^{k}$ be a function of class $C^{1}$ and $(\delta, \epsilon) \in\left(\mathbb{R}_{+}^{*}\right)^{2}$ be such that

1. there exists a compact subset $K$ of $U$ such that $\inf _{U \backslash K}|f|>\delta$,

2. for every y in $U,|f(y)|<\delta \Rightarrow \forall w \in \mathbb{R}^{k},\left|\left(d_{\mid y} f\right)^{*}(w)\right| \geqslant \epsilon|w|$.

Then, for every function $g: U \rightarrow \mathbb{R}^{k}$ of class $C^{1}$ such that $\sup _{U}|g|<\delta$ and $\sup _{U}\|d g\|<\epsilon$, zero is a regular value of $f+g$ and $(f+g)^{-1}(0)$ is compact and isotopic to $f^{-1}(0)$ in $U$.

Proof. The proof is analogous to the one of Lemma 3.4 of [16], since $\left\|(d g)^{*}\right\|=\|d g\|$.

The following Lemma 2.3.3 establishes the existence of peak sections for higher rank vector bundles.

Lemma 2.3.3 (compare Lemma 1.2 of [37]) Let $X$ be a smooth real projective manifold of dimension $n,\left(L, h_{L}\right)$ be a real holomorphic Hermitian line bundle of positive curvature over $X$ and $\left(E, h_{E}\right)$ be a rank $k$ real holomorphic Hermitian vector bundle, with $1 \leqslant k \leqslant n$. Let $x \in \mathbb{R} X,\left(p_{1}, \cdots, p_{n}\right) \in \mathbb{N}^{n}, i \in\{1, \cdots, k\}$ and $p^{\prime}>$ 
$p_{1}+\cdots+p_{n}$. There exists $d_{0} \in \mathbb{N}$ independent of $x$ such that for every $d>d_{0}$, there exists $\sigma \in \mathbb{R} H^{0}\left(X, E \otimes L^{d}\right)$ with the property that $\|\sigma\|_{L^{2}\left(h_{L}\right)}=1$ and if $\left(y_{1}, \cdots, y_{n}\right)$ are local real holomorphic coordinates in the neighbourhood of $x$ and $\left(e_{1}, \cdots e_{k}\right)$ is a local real holomorphic trivialization of $E$ orthonormal at $x$, we can assume that in a neighbourhood of $x$,

$$
\sigma\left(y_{1}, \cdots, y_{n}\right)=\lambda y_{1}^{p_{1}} \cdots y_{n}^{p_{n}} e_{i} \otimes e^{d}\left(1+O\left(d^{-2 p^{\prime}}\right)\right)+O\left(\lambda|y|^{2 p^{\prime}}\right),
$$

where $\lambda^{-2}=\int_{B\left(x, \frac{\log d}{\sqrt{d}}\right)}\left|y_{1}^{p_{1}} \cdots y_{n}^{p_{n}}\right|^{2}\left\|e^{d}\right\|_{h_{L}^{d}}^{2} d V_{h_{L}}$, with $d V_{h_{L}}=\omega^{n} / \int_{X} \omega^{n}$ and where $e$ is a local trivialization of $L$ whose potential $-\log h_{L}(e, e)$ reaches a local minimum at $x$ with Hessian $\pi \omega(., i$.$) .$

Proof. The proof goes along the same lines as the one of Lemma 1.2 of [37]. Let $\eta$ be a cut-off function on $\mathbb{R}$ with $\eta=1$ in a neighbourhood of 0 , and

$$
\psi=\left(n+2 p^{\prime}\right) \eta\left(\frac{d\|z\|^{2}}{\log ^{2} d}\right) \log \left(\frac{d\|z\|^{2}}{\log ^{2} d}\right)
$$

in the coordinates $z$ on $X$. Then, $i \partial \bar{\partial} \psi$ is bounded from below by $-C \omega$, where $C$ is some uniform constant independent of $d$ and $x$. Let $s \in C^{\infty}\left(X, E \otimes L^{d}\right)$ be the real section defined by

$$
s=\eta\left(\frac{d\|z\|^{2}}{\log ^{2} d}\right) y_{1}^{p_{1}} \cdots y_{n}^{p_{n}} e_{i} \otimes e^{d} .
$$

Then, from Theorem 5.1 of [6], for $d$ large enough not depending on $x$, there exists a real section $u \in C^{\infty}\left(X, E \otimes L^{d}\right)$ such that $\bar{\partial} u=\bar{\partial} s$ and satisfying the Hörmander $L^{2}$-estimates

$$
\int_{X}\|u\|_{h_{E, d}}^{2} e^{-\psi} d V_{h_{L}} \leqslant \int_{X}\|\bar{\partial} s\|_{h_{E, d}}^{2} e^{-\psi} d V_{h_{L}}
$$

The presence of the singular weight $e^{-\psi}$ forces the jets of $u$ to vanish up to order $2 p^{\prime}$ at $x$. As in Lemma 1.2 of [37], we conclude that the real holomorphic section $\sigma=(s-u) /\|s-u\|_{L^{2}\left(h_{E, d}\right)}$ satisfies the required properties.

In this first section we will only need peak sections given by Lemma 2.3 .3 with $\sum_{i=1}^{n} p_{i}=0$, whereas in the second one we will need those given with $\sum_{i=1}^{n} p_{i} \leqslant 2$.

Definition 2.3.4 For $i \in\{1, \cdots, k\}$, let $\sigma_{0}^{i}$ be the section given by Lemma 2.3.3 with $p^{\prime}=3$ and $p_{1}=\cdots=p_{n}=0$. Likewise, for every $j \in\{1, \cdots, n\}$, let $\sigma_{j}^{i}$ be a section given by (25) with $p^{\prime}=3, p_{j}=1$ and $p_{l}=0$ for $l \in\{1, \cdots, n\} \backslash\{j\}$. Finally, for every $1 \leqslant l \leqslant m \leqslant n$, let $\sigma_{l m}^{i}$ be a section given by (25) with $p^{\prime}=3, p_{j}=0$ for every $j \in\{1, \cdots, n\} \backslash\{l, m\}$ and $p_{l}=p_{m}=1$ if $l \neq m$, while $p_{l}=2$ otherwise.

The asymptotic values of the constants $\lambda$ in (25) are given by Lemma 2.3.5 (compare Lemma 2.1 of [37]).

Lemma 2.3.5 For every $i \in\{1, \cdots, k\}$, the sections given by Definition 2.3.4 satisfy

$$
\begin{array}{r}
\sigma_{0}^{i} / \sqrt{\delta_{L} d^{n}} \underset{d \rightarrow \infty}{\sim} e_{i} \otimes e^{d}+O\left(\|y\|^{6}\right), \\
\forall j \in\{1, \cdots, n\}, \sigma_{j}^{i} / \sqrt{\pi \delta_{L} d^{n+1}} \underset{d \rightarrow \infty}{\sim} y_{j} e_{i} \otimes e^{d}+O\left(\|y\|^{6}\right), \\
\forall l, m \in\{1, \cdots, n\}, l \neq m, \sigma_{l m}^{i} /\left(\pi \sqrt{\delta_{L} d^{n+2}}\right) \underset{d \rightarrow \infty}{\sim} y_{l} y_{m} e_{i} \otimes e^{d}+O\left(\|y\|^{6}\right), \\
\text { and } \forall l \in\{1, \cdots, n\}, \sigma_{l l}^{i} /\left(\pi \sqrt{\delta_{L} d^{n+2}}\right) \underset{d \rightarrow \infty}{\sim} \frac{1}{\sqrt{2}} y_{l}^{2} e_{i} \otimes e^{d}+O\left(\|y\|^{6}\right) .
\end{array}
$$


Moreover, these sections are asymptotically orthonormal as $d$ grows to infinity, as follows from Lemma 2.3.6.

Lemma 2.3.6 (compare Lemma 3.1 of [37]) For every $x \in \mathbb{R} X$, the sections $\left(\sigma_{j}^{i}\right)_{\substack{1 \leqslant i \leqslant k \\ 0 \leqslant j \leqslant n}}$ and $\left(\sigma_{l m}^{i}\right)_{\substack{1 \leqslant i \leqslant k \\ 1 \leqslant l \leqslant m \leqslant n}}$ given by Definition 2.3.4 have $L^{2}$-norm equal to one and their pairwise scalar product are dominated by a $O\left(d^{-1}\right)$ which does not depend on $x$. Likewise, their scalar products with every section of $\mathbb{R} H^{0}\left(X, E \otimes L^{d}\right)$ of $L^{2}$-norm equal to one and whose 2-jet at $x$ vanishes is dominated by a $O\left(d^{-3 / 2}\right)$ which does not depend on $x$.

Proof. The proof goes along the same lines as the one of Lemma 3.1 of [37].

Lemma 2.3.7 Denote by $v$ the density of $d V_{h_{L}}=\omega^{n} / \int_{X} \omega^{n}$ with respect to the volume form $d x$ chosen in (6), so that $d V_{h_{L}}=v(x) d x$. Then the sections given by Defintion 2.3.4 times $\sqrt{v(x)}$ are still asymptotically orthonormal for (6)).

Proof. This is a direct consequence of Lemmas 2.3.3 and 2.3.6 and the asymptotic concentration of the support of the peak sections near $x$.

Remark 2.3.8 The complex analogues of Lemmas 2.3.3, 2.3.5 and 2.3.6 hold, compare 37 .

\subsection{Proof of Theorem 1.2 .1}

We first compute the expected local $C^{1}$-norm of sections.

Proposition 2.4.1 Let $X$ be a smooth real projective manifold of dimension $n,\left(L, h_{L}\right)$ be a real holomorphic Hermitian line bundle of positive curvature over $X$ and $\left(E, h_{E}\right)$ be a rank $k$ real holomorphic Hermitian vector bundle, with $1 \leqslant k \leqslant n$. We equip $\mathbb{R} X$ with a field of $h_{L}$-trivializations, see $\sqrt{2.3}$. Then, for every positive $R$,

$$
\begin{aligned}
\limsup _{d \rightarrow \infty} \sup _{x \in \mathbb{R} X} \frac{1}{d^{n}} E\left(\sup _{B\left(x, \frac{R}{\sqrt{d}}\right)} \frac{|\sigma|^{2}}{v(x)}\right) \leqslant 6 k \delta_{L} \rho_{R} \text { and } \\
\limsup _{d \rightarrow \infty} \sup _{x \in \mathbb{R} X} \frac{1}{d^{n+1}} E\left(\sup _{B\left(x, \frac{R}{\sqrt{d}}\right)} \frac{\|d \sigma\|_{2}^{2}}{v(x)}\right) \leqslant 6 \pi n k \delta_{L} \rho_{R},
\end{aligned}
$$

where $v$ is given by Lemma 2.3.7 and $\rho_{R}$ is given by (11), see (19) and (20) for the definitions of $|\sigma|$ and $\|d \sigma\|_{2}$.

Note that a global estimate on the sup norm of $L^{2}$ random holomorphic sections is given by Theorem 1.1 of [32].

Proof. The proof goes along the same lines as the proof of Proposition 3.5 of [16]. We first establish from the mean value inequality that for every $x \in \mathbb{R} X, R>0$ and $s>0$,

$$
E\left(\sup _{B\left(x, \frac{R}{\sqrt{d}}\right)}|\sigma|^{2}\right) \leqslant \frac{1}{\operatorname{Vol}\left(B\left(\frac{s}{\sqrt{d}}\right)\right)} \int_{B\left(x, \frac{R+s}{\sqrt{d}}\right)} \mathbb{E}\left(|\sigma|^{2}\right) \psi_{x}^{*} d y
$$


for $d$ large enough not depending on $x$. Then, for every $z \in B\left(x, \frac{R+s}{\sqrt{d}}\right) \cap \mathbb{R} X$, we write $\sigma=\sum_{i=1}^{k} a_{i} \sigma_{0}^{i}+\tau$, where $\tau \in \mathbb{R} H^{0}\left(X, E \otimes L^{d}\right)$ vanishes at $z$ and $\left(\sigma_{0}^{i}\right)_{i=1, \cdots k}$ are the peak sections at $z$ given by Definition 2.3.4. In particular, by Lemma 2.3.5, at the

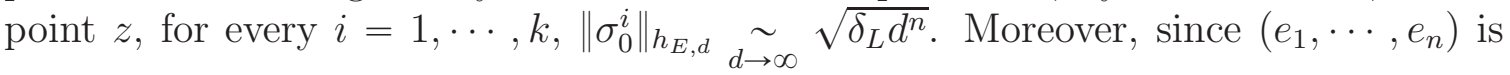
orthonormal at $x$,

$$
\begin{aligned}
\left|\sigma_{0}^{i}(z)\right|^{2} & =\left\|\sigma_{0}^{i}(z)\right\|_{h_{E, d}}^{2}\left(1+O(|z-x|) e^{d \phi(z)}\right. \\
& \leqslant \delta_{L} d^{n} e^{\pi(R+s)^{2}}(1+o(1))
\end{aligned}
$$

from the inequalities (17), where the $o\left(d^{n}\right)$ can be chosen not to depend on $x \in \mathbb{R} X$. Suppose that $d y=d V_{h_{L}}$. Then, by Lemma 2.3.6, the peak sections are asymptotically orthogonal to each other for the scalar product defined by (6), and asymptotically orthogonal to the space of sections $\tau$ vanishing at $x$. We deduce that

$$
\begin{aligned}
\mathbb{E}\left(|\sigma(z)|^{2}\right) & =\mathbb{E}\left(\left|\sum_{i=1}^{k} a_{i} \sigma_{0}^{i}\right|^{2}\right)(1+o(1)) \\
& =\left(\sum_{i=1}^{k}\left|\sigma_{0}^{i}(z)\right|^{2}\right) \frac{1}{\sqrt{\pi}} \int_{\mathbb{R}} a^{2} e^{-a^{2}} d a(1+o(1)) \\
& \leqslant \frac{1}{2} k \delta_{L} d^{n} e^{\pi(R+s)^{2}}(1+o(1)) .
\end{aligned}
$$

When $z \notin B\left(x, \frac{R+s}{\sqrt{d}}\right) \cap \mathbb{R} X$, the space of real sections vanishing at $z$ gets of real codimension $2 k$ in $\mathbb{R} H^{0}\left(X, E \otimes L^{d}\right)$. Let $\left\langle\theta_{1}^{i}, \theta_{2}^{i}, i \in\{1, \cdots, k\}\right\rangle$ be an orthonormal basis of its orthogonal complement. From Remark 2.3.8, for every $i \in\{1, \cdots, k\}$, $j \in\{1,2\}$,

$$
\limsup _{d \rightarrow \infty} \frac{1}{d^{n}}\left|\theta_{j}^{i}(z)\right|^{2} \leqslant 2 \delta_{L} e^{\pi(R+s)^{2}},
$$

an upper bound which does not depend on $z$. We deduce that

$$
\begin{aligned}
\mathbb{E}\left(|\sigma(z)|^{2}\right)= & \int_{\mathbb{R}^{2 k}}\left|\sum_{i=1}^{k}\left(a_{01}^{i} \theta_{1}^{i}(z)+a_{02}^{i} \theta_{2}^{i}(z)\right)\right|^{2} e^{-\sum_{i=1}^{k}\left(a_{01}^{i}\right)^{2}+\left(a_{02}^{i}\right)^{2}} \frac{1}{\pi^{k}} \Pi_{i=1}^{k} d a_{01}^{i} d a_{02}^{i} \\
\leqslant & 2 \delta_{L} d^{n} e^{\pi(R+s)^{2}}(1+o(1)) \sum_{i=1}^{k} \int_{\mathbb{R}^{2}}\left(\left(a_{01}^{i}\right)^{2}+\left(a_{02}^{i}\right)^{2}+2\left|a_{01}^{i}\right|\left|a_{02}^{i}\right|\right) \ldots \\
& \ldots \frac{1}{\pi} e^{-\left(a_{01}^{i}\right)^{2}-\left(a_{02}^{i}\right)^{2}} d a_{01}^{i} d a_{02}^{i} \\
\leqslant & 6 \delta_{L} d^{n} e^{\pi(R+s)^{2}}(1+o(1)) .
\end{aligned}
$$

We deduce the first part of Proposition 2.4 .1 by taking the supremum over $\mathbb{R} X$, choosing $s$ which minimize $g_{R_{(U, P)}}$ and taking the limsup as $d$ grows to infinity.

In general, the Bergman section at $x$ for the $L^{2}$-product (6) associated to the volume form $d x$ is equivalent to the Bergman section $\sigma_{0}$ at $x$ for $d V_{h}$ times $\sqrt{v(x)}$, see Lemma 2.3.7. The same holds true for the $\sigma_{j}$ 's, and the result follows by replacing $\delta_{L}$ with $v(x) \delta_{L}$.

The proof of the second assertion goes along the same lines, see the proof of Proposition 3.5 of [16] (and [31] for similar results). 
As in [16], we then compute the probability of presence of closed affine real algebraic submanifolds, inspired by an approach of Nazarov and Sodin [25], see also [20]. Let $(U, P)$ be a regular pair given by Definition 2.2.1 and $\Sigma=P^{-1}(0) \subset U$. Then, for every $x \in \mathbb{R} X$, we set $B_{d}=B\left(x, \frac{R_{(U, P)}}{\sqrt{d}}\right) \cap \mathbb{R} X$, see (23) , and denote by $\operatorname{Prob}_{x, \Sigma}\left(E \otimes L^{d}\right)$ the probability that $\sigma \in \mathbb{R} H^{0}\left(X, E \otimes L^{d}\right)$ has the property that $\sigma^{-1}(0) \cap B_{d}$ contains a closed submanifold $\Sigma^{\prime}$ such that the pair $\left(B_{d}, \Sigma^{\prime}\right)$ be diffeomorphic to $\left(\mathbb{R}^{n}, \Sigma\right)$. That is,

$$
\operatorname{Prob}_{x, \Sigma}\left(E \otimes L^{d}\right)=\mu_{\mathbb{R}}\left\{\sigma \in \mathbb{R} H^{0}\left(X, E \otimes L^{d}\right) \mid\left(\sigma^{-1}(0) \cap B_{d}\right) \supset \Sigma^{\prime},\left(B_{d}, \Sigma^{\prime}\right) \sim\left(\mathbb{R}^{n}, \Sigma\right)\right\} .
$$

We then set $\operatorname{Prob}_{\Sigma}\left(E \otimes L^{d}\right)=\inf _{x \in \mathbb{R} X} \operatorname{Prob}_{x, \Sigma}\left(E \otimes L^{d}\right)$.

Proposition 2.4.2 Let $X$ be a smooth real projective manifold of dimension $n,\left(L, h_{L}\right)$ be a real holomorphic Hermitian line bundle of positive curvature over $X$ and $\left(E, h_{E}\right)$ be a rank $k$ real holomorphic Hermitian vector bundle, with $1 \leqslant k \leqslant n$. Let $(U, P)$ be a regular pair given by Definition 2.2.1 and $\Sigma=P^{-1}(0) \subset U$. Then,

$$
\liminf _{d \rightarrow \infty} \operatorname{Prob}_{\Sigma}\left(E \otimes L^{d}\right) \geqslant m_{\tau_{(U, P)}},
$$

see (15).

Proof. The proof is the same as the one of Proposition 3.6 of [16] and is not reproduced here.

The proof of Theorem 1.2.1 (resp. Corollary 2.1.2) then just goes along the same lines as the one of Theorem 1.2 (resp. Corollary 1.3) of [16].

\subsection{Proof of Theorem 2.1.1}

Let $(U, P)$ be a regular pair given by Definition 2.2.1. For every $d>0$, let $\Lambda_{d}$ be a maximal subset of $\mathbb{R} X$ with the property that two distinct points of $\Lambda_{d}$ are at distance greater than $\frac{2 R_{(U, P)}}{\sqrt{d}}$. The balls centered at points of $\Lambda_{d}$ and of radius $\frac{R_{(U, P)}}{\sqrt{d}}$ are disjoints, whereas the ones of radius $\frac{2 R_{(U, P)}}{\sqrt{d}}$ cover $\mathbb{R} X$. Note that if we use the local flat metric given by a trivial $h_{L}$-trivialization, then the associated lattice has asymptotically the same number of balls than $\Lambda_{d}$ as $d$ grows to infinity, so we can suppose from now on that the balls are defined for this local metric. For every $\sigma \in \mathbb{R} H^{0}\left(X, E \otimes L^{d}\right)$, denote by $N_{\Sigma}\left(\Lambda_{d}, \sigma\right)$ the number of $x \in \Lambda_{d}$ such that the ball $B_{d}=B\left(x, \frac{R_{(U, P)}}{\sqrt{d}}\right) \cap \mathbb{R} X$ contains a codimension $k$ submanifold $\Sigma^{\prime}$ with $\Sigma^{\prime} \subset \sigma^{-1}(0)$ and $\left(B_{d}, \Sigma^{\prime}\right)$ diffeomorphic to $\left(\mathbb{R}^{n}, \Sigma\right)$. By definition of $N_{\Sigma}(\sigma), N_{\Sigma}\left(\Lambda_{d}, \sigma\right) \leqslant N_{\Sigma}(\sigma)$, see $§ 1.2$, while from Proposition 2.4.2, for every $0<\epsilon<1$,

$$
\begin{aligned}
\left|\Lambda_{d}\right| m_{\tau_{(U, P)}} \leqslant & \sum_{x \in \Lambda_{d}} \operatorname{Prob}_{x, \Sigma}\left(E \otimes L^{d}\right) \\
\leqslant & \sum_{j=1}^{\left|\Lambda_{d}\right|} j \mu_{\mathbb{R}}\left\{\sigma \mid N_{\Sigma}\left(\Lambda_{d}, \sigma\right)=j\right\} \\
\leqslant & \epsilon m_{\tau_{(U, P)}}\left|\Lambda_{d}\right| \mu_{\mathbb{R}}\left\{\sigma \mid N_{\Sigma}\left(\Lambda_{d}, \sigma\right) \leqslant \epsilon m_{\left.\tau_{(U, P)}\left|\Lambda_{d}\right|\right\}}\right. \\
& +\left|\Lambda_{d}\right| \mu_{\mathbb{R}}\left\{\sigma\left|N_{\Sigma}\left(\Lambda_{d}, \sigma\right) \geqslant \epsilon m_{\tau_{(U, P)}}\right| \Lambda_{d} \mid\right\} .
\end{aligned}
$$


We deduce that

$$
(1-\epsilon) m_{\tau_{(U, P)}} \leqslant \mu_{\mathbb{R}}\left\{\sigma\left|N_{\Sigma}(\sigma) \geqslant \epsilon m_{\tau_{(U, P)}}\right| \Lambda_{d} \mid\right\}
$$

and the result follows by choosing a sequence $\left(U_{p}, P_{p}\right)_{p} \in \mathcal{I}_{\Sigma}$ such that

$$
\lim _{p \rightarrow \infty} m_{\tau_{\left(U_{p}, P_{p}\right)}}\left|\Lambda_{d}\right|=c_{\Sigma} V o l_{h_{L}}(\mathbb{R} X) \sqrt{d}^{n}
$$

see (14).

\subsection{Proof of Corollary 1.2 .2}

In this paragraph, for every positive integer $p, S^{p}$ denotes the unit sphere in $\mathbb{R}^{p+1}$. Corollary 1.2.2 is a consequence of Theorem 1.2.1 and the following Propositions 2.6.1 and 2.6 .3 .

Proposition 2.6.1 For every $1 \leqslant k \leqslant n, c_{S^{n-k}} \geqslant \exp \left(-e^{54+5 n}\right)$.

Recall the following.

Lemma 2.6.2 (Lemma 2.2 of [16]) If $P=\sum_{\left(i_{1}, \cdots, i_{n}\right) \in \mathbb{N}^{n}} a_{i_{1}, \cdots, i_{n}} z_{1}^{i_{1}} \cdots z_{n}^{i_{n}} \in \mathbb{R}\left[z_{1}, \cdots, z_{n}\right]$, then

$$
\|P\|_{L^{2}}^{2}=\int_{\mathbb{C}^{n}}|P(z)|^{2} e^{-\pi|z|^{2}} d z=\sum_{\left(i_{1}, \cdots, i_{n}\right) \in \mathbb{N}^{n}}\left|a_{i_{1}, \cdots, i_{n}}\right|^{2} \frac{i_{1} ! \cdots i_{n} !}{\pi^{i_{1}+\cdots+i_{n}}} .
$$

Proof of Proposition 2.6.1. For every $n>0$, we set $P_{k}\left(x_{1}, \cdots, x_{n}\right)=$ $\sum_{j=k}^{n} x_{j}^{2}-1$. For every $x \in \mathbb{R}^{n}$ and $\delta>0$,

$$
\left|P_{k}(x)\right|<\delta \Leftrightarrow 1-\delta<\sum_{i=k}^{n} x_{i}^{2}<1+\delta \Rightarrow\left\|d_{\mid x} P_{k}\right\|_{2}^{2}=4 \sum_{i=k}^{n} x_{i}^{2}>4(1-\delta) .
$$

Moreover from Lemma 2.6.2,

$$
\left\|P_{k}\right\|_{L^{2}}^{2}=1+\frac{2(n-k+1)}{\pi^{2}} \leqslant n-k+2 .
$$

Now set $P_{S}=\left(P_{1}, \cdots, P_{k}\right)$ with $P_{j}(x)=x_{j}$ for $1 \leqslant j \leqslant k-1$, so that

$$
\left\|P_{S}\right\|_{L_{2}}^{2} \leqslant(k-1) / \pi+(n-k+2) \leqslant n+1 \leqslant 2 n .
$$

Since for every $w=\left(w_{1}, \cdots, w_{k}\right) \in \mathbb{R}^{k}$ and every $x \in \mathbb{R}^{n}$,

$$
\left|d_{\mid x} P_{S}^{*}(w)\right|^{2}=\sum_{i=1}^{k-1} w_{i}^{2}+w_{k}^{2}\left\|d_{\mid x} P_{k}\right\|_{2}^{2}
$$

we get that $\left\|d_{\mid x} P_{S}^{*}\right\|^{2} \geqslant \min (1,4(1-\delta))$ if $\left|P_{k}(x)\right|<\delta$. Choose

$$
U_{S}=\left\{\left(x_{1}, \cdots, x_{n}\right) \in \mathbb{R}^{n} \mid \sum_{j=1}^{n} x_{j}^{2}<4\right\}
$$


Then if $0<\delta<1$,

$$
K_{\delta}=\left\{x \in U_{S} \mid 1-\delta \leqslant \sum_{i=k}^{n} x_{i}^{2} \leqslant 1+\delta \text { and } \sum_{i=1}^{k-1} x_{k}^{2} \leqslant 1-\frac{1}{2}(1+\delta)^{2}\right\}
$$

is compact in $U_{S}$ and taking $R_{\left(U_{S}, P_{S}\right)}^{2}=4$, we see that the pair $\left(U_{S}, P_{S}\right)$ is regular in the sense of Definition 2.2.1. The submanifold $P_{S}^{-1}(0) \subset U_{S}$ is isotopic in $\mathbb{R}^{n}$ to the unit sphere $S^{n-k}$. We deduce that $(3 / 4,1) \in \mathcal{T}_{\left(U_{S}, P_{S}\right)}$. From (10) and (13) we deduce

$$
\tau_{\left(U_{S}, P_{S}\right)} \leqslant 24 k 4^{n} e^{16 \pi} 2 n(2+\pi n) \leqslant e^{53+5 n} .
$$

The estimate $c_{S^{n-1}} \geqslant \exp \left(-e^{54+5 n}\right)$ follows then from (16) .

Proposition 2.6.3 For every $1 \leqslant k \leqslant n$ and every $0 \leqslant i \leqslant n-k, c_{S^{i} \times S^{n-i-k}} \geqslant$ $\exp \left(-e^{82+6 n}\right)$.

Proof. For every $1 \leqslant k \leqslant n$ and every $0 \leqslant i \leqslant n-k$, we set

$$
Q_{k}\left(\left(x_{1}, \cdots, x_{i+1}\right),\left(y_{1}, \cdots, y_{n-i-1}\right)\right)=\left(|x|^{2}-2\right)^{2}+\sum_{j=1}^{n-k-i} y_{j}^{2}-1 .
$$

For every $(x, y) \in \mathbb{R}^{i+1} \times \mathbb{R}^{n-i-1}$ and $0<\delta<1 / 2$,

$$
\begin{aligned}
\left|Q_{k}(x, y)\right|<\delta & \Leftrightarrow 1-\delta<\left(|x|^{2}-2\right)^{2}+\sum_{j=1}^{n-k-i} y_{j}^{2}<1+\delta \\
& \Rightarrow\left\|d_{\mid(x, y)} Q_{k}\right\|_{2}^{2}=4 \sum_{j=1}^{n-k-i} y_{j}^{2}+16|x|^{2}\left(|x|^{2}-2\right)^{2}
\end{aligned}
$$

with $|x|^{2}>2-\sqrt{1+\delta}>1 / 2$. Thus $\left\|d_{\mid(x, y)} Q_{k}\right\|_{2}^{2}>4(1-\delta)$, compare Lemma 2.6 of [16]. Moreover from Lemma 2.6.2, $\left\|Q_{k}\right\|_{L^{2}}^{2} \leqslant 13 n^{2}$, compare $\S 2.3 .2$ of [16]. Now set $Q=\left(Q_{1}, \cdots, Q_{k}\right)$ with $Q_{j}(x, y)=y_{n-i-j}$ for $1 \leqslant j \leqslant k-1$, so that

$$
\|Q\|_{L_{2}}^{2} \leqslant(k-1) / \pi+13 n^{2} \leqslant 13(n+1)^{2} .
$$

For every $w=\left(w_{1}, \cdots, w_{k}\right) \in \mathbb{R}^{k}$ and every $(x, y) \in \mathbb{R}^{i+1} \times \mathbb{R}^{n-i-1}$,

$$
\begin{aligned}
\left|d_{\mid(x, y)} Q^{*}(w)\right|^{2} & =\sum_{i=1}^{k-1} w_{i}^{2}+w_{k}^{2}\left\|d_{\mid(x, y)} Q_{k}\right\|_{2}^{2} \\
& >\min (1,4(1-\delta))|w|^{2}
\end{aligned}
$$

if $\left|Q_{k}(x, y)\right| \leqslant \delta<1 / 2$. We choose

$$
\begin{gathered}
U=\left\{(x, y) \in \mathbb{R}^{i+1} \times\left.\mathbb{R}^{n-i-1}|| x\right|^{2}+|y|^{2}<6\right\}, \\
K_{\delta}=\left\{(x, y) \in U \mid 1-\delta \leqslant\left(|x|^{2}-2\right)^{2}+\sum_{j=1}^{n-k-i} y_{j}^{2} \leqslant 1+\delta \text { and } \sum_{j=1}^{k-1} y_{n-i-j}^{2} \leqslant 1-\delta\right\},
\end{gathered}
$$


and $R_{(U, Q)}^{2}=6$. The pair $(U, Q)$ is regular in the sense of Definition 2.2.1 and $Q^{-1}(0) \subset U$ is isotopic in $\mathbb{R}^{n}$ to the product $S^{i} \times S^{n-i-k}$ of unit spheres in $\mathbb{R}^{i+1}$ and $\mathbb{R}^{n-i-k+1}$. We deduce that for every positive $\epsilon,(1 / 2-\epsilon, 1) \in \mathcal{T}_{(U, Q)}$ and from (10) and (13) that

$$
\tau_{(U, Q)} \leqslant 24 k 4^{n} e^{24 \pi} 13(n+1)^{2}(4+\pi n) \leqslant e^{81+6 n} .
$$

The estimate $c_{S^{i} \times S^{n-i-k}} \geqslant \exp \left(-e^{82+6 n}\right)$ follows then from (16).

\section{Upper estimates for the expected Betti numbers}

\subsection{Statement of the results}

For every $1 \leqslant k \leqslant n$, we denote by $\operatorname{Gr}(k-1, n-1)$ the Grassmann manifold of $(k-1)$-dimensional linear subspaces of $\mathbb{R}^{n-1}$. The tangent space of $\operatorname{Gr}(k-1, n-1)$ at every $H \in \operatorname{Gr}(k-1, n-1)$ is canonically isomorphic to the space of linear maps $L\left(H, H^{\perp}\right)$ from $H$ to its orthogonal $H^{\perp}$ and we equip it with the norm

$$
A \in L\left(H, H^{\perp}\right) \mapsto\|A\|_{2}=\sqrt{\operatorname{Tr}\left(A^{*} A\right)} \in \mathbb{R}^{+} .
$$

The total volume of $\operatorname{Gr}(k-1, n-1)$ for this Riemannian metric is denoted by $\operatorname{Vol}(\operatorname{Gr}(k-1, n-1))$ and we set

$$
V_{k-1, n-1}=\frac{1}{\sqrt{\pi}^{(k-1)(n-k)}} \operatorname{Vol}(\operatorname{Gr}(k-1, n-1))
$$

its volume for the rescaled metric $A \in L\left(H, H^{\perp}\right) \mapsto \frac{1}{\sqrt{\pi}}\|A\|_{2}$. Likewise, we equip $M_{k-1}(\mathbb{R})$ with the Euclidean norm $A \in M_{k-1}(\mathbb{R}) \mapsto\|A\|_{2}=\sqrt{\operatorname{Tr}\left(A^{*} A\right)}$ and set $d \mu(A)=\frac{1}{\pi^{k-1}} e^{-\|A\|_{2}^{2}} d A$ the associated Gaussian measure on $M_{k-1}(\mathbb{R})$. Then, we set

$$
E_{k-1}\left(|\operatorname{det}|^{n-k+2}\right)=\int_{M_{k-1(\mathbb{R})}}|\operatorname{det} A|^{n-k+2} d \mu(A) .
$$

Remark 3.1.1 1. The orthogonal group $O_{n-1}(\mathbb{R})$ acts transitively on the Grassmannian $\operatorname{Gr}(k-1, n-1)$ with fixators isomorphic to $O_{k-1}(\mathbb{R}) \times O_{n-k}(\mathbb{R})$. We deduce that

$$
\begin{aligned}
\operatorname{Vol}(\operatorname{Gr}(k-1, n-1)) & =\operatorname{Vol}\left(O_{n-1}(\mathbb{R})\right) /\left(\operatorname{Vol}\left(O_{k-1}(\mathbb{R})\right) \times \operatorname{Vol}\left(O_{n-k}(\mathbb{R})\right)\right) \\
& =\left(\begin{array}{c}
n-1 \\
k-1
\end{array}\right) \sqrt{\pi}^{(k-1)(n-k)} \frac{\prod_{j=1}^{k-1} \Gamma(1+j / 2)}{\prod_{j=n-k+1}^{n-1} \Gamma(1+j / 2)},
\end{aligned}
$$

where $\Gamma$ denotes the Gamma function of Euler, see for example Lemma 3.4 of 14.

2. From formula (15.4.12) of [23] follows that

$$
E_{k-1}\left(|\operatorname{det}|^{n-k+2}\right)=\prod_{j=1}^{k-1} \frac{\Gamma\left(\frac{n-k+2+j}{2}\right)}{\Gamma\left(\frac{j}{2}\right)},
$$

so that $V_{k-1, n-1} E_{k-1}\left(|\operatorname{det}|^{n-k+2}\right)=\frac{(n-1) !}{(n-k) ! 2^{k-1}}$. 
We now keep the framework of $\$ 2.1$. Let us denote, for every $i \in\{0, \cdots, n-k\}$, by $b_{i}\left(\mathbb{R} C_{\sigma}, \mathbb{R}\right)=\operatorname{dim} H_{i}\left(\mathbb{R} C_{\sigma}, \mathbb{R}\right)$ the $i$-th Betti number of $\mathbb{R} C_{\sigma}$ and by

$$
m_{i}\left(\mathbb{R} C_{\sigma}\right)=\inf _{f \text { Morse on } \mathbb{R} C_{\sigma}}\left|\operatorname{Crit}_{i}(f)\right|
$$

its $i$-th Morse number, where $\left|\operatorname{Crit}_{i}(f)\right|$ denotes the number of critical points of index $i$ of $f$. We then denote by

$$
\mathbb{E}\left(b_{i}\right)=\int_{\mathbb{R} H^{0}\left(X, E \otimes L^{d}\right) \backslash \mathbb{R} \Delta_{d}} b_{i}\left(\mathbb{R} C_{\sigma}, \mathbb{R}\right) d \mu_{\mathbb{R}}(\sigma)
$$

and

$$
\mathbb{E}\left(m_{i}\right)=\int_{\mathbb{R} H^{0}\left(X, E \otimes L^{d}\right) \backslash \mathbb{R} \Delta_{d}} m_{i}\left(\mathbb{R} C_{\sigma}\right) d \mu_{\mathbb{R}}(\sigma)
$$

their expected values. The aim of $\oint 3$ is to prove the following Theorem $[3.1 .2$, see (1) for the definition of $e_{\mathbb{R}}(i, n-k-i)$.

Theorem 3.1.2 Let $X$ be a smooth real projective manifold of dimension $n,\left(L, h_{L}\right)$ be a real holomorphic Hermitian line bundle of positive curvature over $X$ and $\left(E, h_{E}\right)$ be a rank $k$ real holomorphic Hermitian vector bundle, with $1 \leqslant k \leqslant n-1$. Then, for every $0 \leqslant i \leqslant n-k$,

$$
\limsup _{d \rightarrow \infty} \frac{1}{\sqrt{d}^{n}} \mathbb{E}\left(m_{i}\right) \leqslant \frac{1}{\Gamma\left(\frac{k}{2}\right)} V_{k-1, n-1} E_{k-1}\left(|\operatorname{det}|^{n-k+2}\right) e_{\mathbb{R}}(i, n-k-i) \operatorname{Vol}_{h_{L}}(\mathbb{R} X) .
$$

Note that the case $k=n$ is covered by Theorems 1.1.1 and 3.1.3. When $k=1$ and $E=\mathcal{O}_{X}, \operatorname{Vol}_{F S}\left(\mathbb{R} P^{k}\right)=\sqrt{\pi}$, see Remark 2.14 of [14, so that Theorem 3.1.2 reduces to Theorem 1.0.1 of [14] in this case. The proof of Theorem 3.1.2 actually goes along the same lines as the one of Theorem 1.1 of [14]. The strategy goes as follows. We fix a Morse function $p: \mathbb{R} X \rightarrow \mathbb{R}$. Then, almost surely on $\sigma \in \mathbb{R} H^{0}\left(X, E \otimes L^{d}\right)$, the restriction of $p$ to $\mathbb{R} C_{\sigma}$ is itself a Morse function. For $i \in\{0, \cdots, n-k\}$, we denote by $\operatorname{Crit}_{i}\left(p_{\mid \mathbb{R} C_{\sigma}}\right)$ the set of critical points of index $i$ of this restriction and set

$$
\nu_{i}\left(\mathbb{R} C_{\sigma}\right)=\frac{1}{\sqrt{d}^{n}} \sum_{x \in \operatorname{Crit}_{i}\left(p_{\mid \mathbb{R} C_{\sigma}}\right)} \delta_{x}
$$

if $n>k$ and $\nu_{0}\left(\mathbb{R} C_{\sigma}\right)=\frac{1}{\sqrt{d}^{n}} \sum_{x \in \mathbb{R} C_{\sigma}} \delta_{x}$ if $k=n$. We then set

$$
\mathbb{E}\left(\nu_{i}\right)=\int_{\mathbb{R} H^{0}\left(X, E \otimes L^{d}\right)} \nu_{i}\left(\mathbb{R} C_{\sigma}\right) d \mu_{\mathbb{R}}(\sigma)
$$

and prove the following equidistribution result (compare Theorem 1.2 of [14]).

Theorem 3.1.3 Under the hypotheses of Theorem 3.1.2, let $p: \mathbb{R} X \rightarrow \mathbb{R}$ be a Morse function. Then, for every $i \in\{0, \cdots, n-k\}$, the measure $\mathbb{E}\left(\nu_{i}\right)$ weakly converges to

$$
\frac{1}{\Gamma\left(\frac{k}{2}\right)} V_{k-1, n-1} E_{k-1}\left(|\operatorname{det}|^{n-k+2}\right) e_{\mathbb{R}}(i, n-k-i) d v o l_{h_{L}}
$$

as d grows to infinity. When $k=n, \mathbb{E}\left(\nu_{0}\right)$ converges weakly to $\frac{1}{\sqrt{\pi}} \Gamma\left(\frac{n+1}{2}\right) d_{\text {vol }} h_{h_{L}}$. 
In Theorem $3.1 .3 d v o l_{h_{L}}$ denotes the Lebesgue measure of $\mathbb{R} X$ induced by the Kähler metric. Theorem 3.1.2 is deduced from Theorem 3.1.3 by integration of 1 over $\mathbb{R} X$. The next paragraphs are devoted to the proof of Theorem 3.1.3,

Proof of Theorem 1.1.1. It follows from Theorem 3.1.2, the Morse inequalities, Remark 3.1.1 and the computation $\operatorname{Vol}_{F S} \mathbb{R} P^{n}=\sqrt{\pi} / \Gamma\left(\frac{n+1}{2}\right)$ (see Remark 2.14 of [14]) when $k \leqslant n-1$ and from Theorem 3.1 .3 when $k=n$.

\subsection{Incidence varieties}

Under the hypotheses of Theorem 3.1.3, we set

$$
\mathbb{R} \Delta_{p}^{d}=\left\{\sigma \in \mathbb{R} H^{0}\left(X, E \otimes L^{d}\right) \mid \sigma \in \mathbb{R} \Delta_{d} \text { or } p_{\mid \mathbb{R} C_{\sigma}} \text { is not Morse }\right\}
$$

and

$$
\mathcal{I}_{i}=\left\{(\sigma, x) \in\left(\mathbb{R} H^{0}\left(X, E \otimes L^{d}\right) \backslash \mathbb{R} \Delta_{p}^{d}\right) \times(\mathbb{R} X \backslash \operatorname{Crit}(p)) \mid x \in \operatorname{Crit}_{i}\left(p_{\mid \mathbb{R} C_{\sigma}}\right)\right\} .
$$

We set

$$
\begin{aligned}
& \pi_{1}:(\sigma, x) \in \mathcal{I}_{i} \mapsto \sigma \in \mathbb{R} H^{0}\left(X, E \otimes L^{d}\right) \text { and } \\
& \pi_{2}:(\sigma, x) \in \mathcal{I}_{i} \mapsto x \in \mathbb{R} X .
\end{aligned}
$$

Then, for every $\left(\sigma_{0}, x_{0}\right) \in\left(\left(\mathbb{R} H^{0}\left(X, E \otimes L^{d}\right) \backslash \mathbb{R} \Delta_{p}^{d}\right) \times(\mathbb{R} X \backslash C r i t(p))\right), \pi_{1}$ is invertible in a neighbourhood $\mathbb{R} U$ of $\sigma_{0}$, defining an evaluation map at the critical point

$$
e v_{\left(\sigma_{0}, x_{0}\right)}: \sigma \in \mathbb{R} U \mapsto \pi_{2} \circ \pi_{1}^{-1}(\sigma)=x \in \operatorname{Crit}_{i}\left(p_{\mid \mathbb{R} C_{\sigma}}\right) \cap \mathbb{R} V
$$

where $\mathbb{R} V$ denotes a neighbourhood of $x_{0}$ in $\mathbb{R} X$, compare $\S 2.4 .2$ of [14]. We denote by $d_{\mid \sigma_{0}} e v_{\left(\sigma_{0}, x_{0}\right)}^{\perp}$ the restriction of its differential map $d_{\mid \sigma_{0}} e v_{\left(\sigma_{0}, x_{0}\right)}$ at $\sigma_{0}$ to the orthogonal complement of $\pi_{1}\left(\pi_{2}^{-1}\left(x_{0}\right)\right)$ in $\mathbb{R} H^{0}\left(X, E \otimes L^{d}\right)$.

Proposition 3.2.1 Under the hypotheses of Theorem 3.1.3,

$$
\mathbb{E}\left(\nu_{i}\right)=\frac{1}{\sqrt{d}^{n}}\left(\pi_{2}\right)_{*}\left(\pi_{1}^{*} d \mu_{\mathbb{R}}\right) .
$$

Moreover, at every point $x \in \mathbb{R} X \backslash \operatorname{Crit}(p)$,

$$
\left(\pi_{2}\right)_{*}\left(\pi_{1}^{*} d \mu_{\mathbb{R}}\right)_{\mid x}=\frac{1}{\sqrt{\pi}^{n}} \int_{\pi_{1}\left(\pi_{2}^{-1}(x)\right)}\left|\operatorname{det} d_{\mid \sigma} e v_{(\sigma, x)}^{\perp}\right|^{-1} d \mu_{\mathbb{R}}(\sigma) d v o l_{h_{L}} .
$$

Proof. The proof is the same as in the one of Proposition 2.10 of [14] and is not reproduced here.

Fix $x \in \mathbb{R} X \backslash \operatorname{Crit}(p)$. Then $\pi_{1}\left(\pi_{2}^{-1}(x)\right)$ is open in a subspace of $\mathbb{R} H^{0}\left(X, E \otimes L^{d}\right)$. Namely,

$$
\begin{aligned}
\pi_{1}\left(\pi_{2}^{-1}(x)\right)= & \left\{\sigma \in \mathbb{R} H^{0}\left(X, E \otimes L^{d}\right) \backslash \mathbb{R} \Delta_{p}^{d} \mid \sigma(x)=0\right. \text { and } \\
& \left.\exists \lambda \in \mathbb{R}\left(E \otimes L^{d}\right)_{\mid x}^{*}, \lambda \circ \nabla_{\mid x} \sigma=d_{\mid x} p\right\}
\end{aligned}
$$


where $\mathbb{R}\left(\left(E \otimes L^{d}\right)_{\mid x}^{*}\right)$ is the real part of the fibre $\left(E \otimes L^{d}\right)_{\mid x}^{*}$. We deduce a well-defined map

$$
\begin{aligned}
\rho_{x}: \pi_{1}\left(\pi_{2}^{-1}(x)\right) & \rightarrow \operatorname{Gr}\left(n-k, \operatorname{ker} d_{\mid x} p\right) \times\left(\mathbb{R}\left(E \otimes L^{d}\right)_{\mid x}^{*} \backslash\{0\}\right) \\
\sigma & \mapsto\left(\operatorname{ker} \nabla_{\mid x} \sigma, \lambda\right) .
\end{aligned}
$$

For every $\sigma \in \mathbb{R} H^{0}\left(X, E \otimes L^{d}\right) \backslash \mathbb{R} \Delta_{p}^{d}$, the tangent space of $\pi_{1}\left(\pi_{2}^{-1}(x)\right)$ at $\sigma$ reads

$$
\begin{aligned}
T_{\sigma} \pi_{1}\left(\pi_{2}^{-1}(x)\right)= & \left\{\dot{\sigma} \in \mathbb{R} H^{0}\left(X, E \otimes L^{d}\right) \mid \dot{\sigma}(x)=0\right. \text { and } \\
& \left.\exists \dot{\lambda} \in \mathbb{R}\left(E \otimes L^{d}\right)_{\mid x}^{*} \mid \dot{\lambda} \circ \nabla_{\mid x} \sigma+\lambda \circ \nabla \dot{\sigma}_{\mid x}=0\right\} .
\end{aligned}
$$

Likewise, for every $\lambda \in \mathbb{R}\left(E \otimes L^{d}\right)_{\mid x}^{*} \backslash\{0\}$, the tangent space of $\rho_{x}^{-1}\left(\operatorname{Gr}\left(n-k\right.\right.$, ker $\left.d_{\mid x} p\right) \times$ $\{\lambda\})$ at $\sigma$ reads

$T_{\sigma} \rho_{x}^{-1}\left(\operatorname{Gr}\left(n-k, \operatorname{ker} d_{\mid x} p\right) \times\{\lambda\}\right)=\left\{\dot{\sigma} \in \mathbb{R} H^{0}\left(X, E \otimes L^{d}\right) \mid \dot{\sigma}(x)=0\right.$ and $\left.\lambda \circ \nabla_{\mid x} \dot{\sigma}=0\right\}$.

Finally, for every $K \in \operatorname{Gr}\left(n-k\right.$, $\left.\operatorname{ker} d_{\mid x} p\right)$, the tangent space of $\rho_{x}^{-1}(K, \lambda)$ at $\sigma$ reads

$$
T_{\sigma} \rho_{x}^{-1}(K, \lambda)=\left\{\dot{\sigma} \in \mathbb{R} H^{0}\left(X, E \otimes L^{d}\right) \mid \dot{\sigma}(x)=0, \nabla_{\mid x} \dot{\sigma}_{\mid K}=0 \text { and } \lambda \circ \nabla_{\mid x} \dot{\sigma}=0\right\} .
$$

Let us choose local real holomorphic coordinates $\left(x_{1}, \cdots, x_{n}\right)$ of $X$ near $x$ such that $\left(\partial / \partial x_{1}, \cdots, \partial / \partial x_{n}\right)$ be orthonormal at $x$, with $d_{\mid x} p$ being colinear to $d x_{1}$ and such that $K=\operatorname{ker} \nabla_{\mid x} \sigma=\left\langle\partial / \partial x_{k+1}, \cdots, \partial / \partial x_{n}\right\rangle$. Let us choose a local real holomorphic trivialization $\left(e_{1}, \cdots, e_{k}\right)$ of $E$ near $x$ that is orthonormal at $x$ and be such that ker $\lambda_{\mid x}=\left\langle e_{2} \otimes e^{d}, \cdots, e_{k} \otimes e^{d}\right\rangle_{\mid x}$. For $d$ large enough, we define the following subspaces of $\mathbb{R} H^{0}\left(X, E \otimes L^{d}\right)$ :

$$
\begin{aligned}
H_{x} & =\left\langle\left(\sigma_{0}^{i}\right)_{1 \leqslant i \leqslant k},\left(\sigma_{j}^{1}\right)_{k+1 \leqslant j \leqslant n}\right\rangle \\
H_{\lambda} & =\left\langle\left(\sigma_{j}^{1}\right)_{1 \leqslant j \leqslant k}\right\rangle \\
H_{K} & =\left\langle\left(\sigma_{j}^{i}\right)_{\substack{2 \leqslant i \leqslant k \\
k+1 \leqslant j \leqslant n}}\right\rangle,
\end{aligned}
$$

where the sections $\left(\sigma_{0}^{i}\right)_{1 \leqslant i \leqslant k}$ and $\left(\sigma_{j}^{i}\right)_{\substack{1 \leqslant i \leqslant k \\ 1 \leqslant j \leqslant n}}$ of $\mathbb{R} H^{0}\left(X, E \otimes L^{d}\right)$ are given by Lemma 2.3 .3 and Definition 2.3.4.

$H_{K}$ is a complement of $T_{\sigma} \rho_{x}^{-1}(K, \lambda)$ in $T_{\sigma} \rho_{x}^{-1}\left(\operatorname{Gr}\left(n-k, \operatorname{ker} d_{\mid x} p\right) \times\{\lambda\}\right), H_{\lambda}$ is a complement of $T_{\sigma} \rho_{x}^{-1}\left(\operatorname{Gr}\left(n-k\right.\right.$, $\left.\left.\operatorname{ker} d_{\mid x} p\right) \times\{\lambda\}\right)$ in $T_{\sigma} \pi_{1}\left(\pi_{2}^{-1}(x)\right)$ and $H_{x}$ is a complement of $T_{\sigma} \pi_{1}\left(\pi_{2}^{-1}(x)\right)$ in $\mathbb{R} H^{0}\left(X, E \otimes L^{d}\right)$. Then, from Lemmas 2.3.6 and 2.3.7, up to a uniform rescaling by $\sqrt{v(x)}$, these complements are asymptotically orthogonal and their given basis orthonormal. Hence, we can assume from now on that $v=1$.

Lemma 3.2.2 Under the hypotheses of Theorem 3.1.3, let $(\sigma, x) \in \mathcal{I}_{i}$ and $\lambda \in \mathbb{R}(E \otimes$ $\left.L^{d}\right)_{\mid x}^{*} \backslash\{0\}$ such that $\lambda \circ \nabla_{\mid x} \sigma=d_{\mid x} p$. Then, $\lambda \circ \nabla^{2} \sigma_{\mid K_{x}}=\nabla^{2}\left(p_{\left.\mid \mathbb{R} C_{\sigma}\right)_{\mid x}}\right.$, so that the quadratic form $\lambda \circ \nabla^{2} \sigma_{\mid K_{x}}$ is non-degenerated of index $i$.

Proof. The proof is similar to the one of Lemma 2.9 of [14]. 


\subsection{Computation of the Jacobian determinants}

\subsubsection{Jacobian determinant of $\rho_{x}$}

Under the hypotheses of Theorem 3.1.3, let $(\sigma, x) \in \mathcal{I}_{i}$. We set $(K, \lambda)=\rho_{x}(\sigma)$ and denote by $d_{\mid \sigma} \rho_{x}^{H}$ the restriction of $d_{\mid \sigma} \rho_{x}$ to $H_{K} \oplus H_{\lambda}$. We then $\operatorname{denote}$ by $\operatorname{det}\left(d_{\mid \sigma} \rho_{x}^{H}\right)$ the Jacobian determinant of $d_{\mid \sigma} \rho_{x}^{H}$ computed in the given basis of $H_{\lambda}$ and $H_{K}$, see (38), (39) and in orthonormal basis of $T_{K} \operatorname{Gr}\left(n-k, \operatorname{ker} d_{\mid x} p\right) \times \mathbb{R}\left(E \otimes L^{d}\right)_{\mid x}^{*}$. By assumption, the operator $\nabla_{\mid x} \sigma$ does not depend on the choice of a connection $\nabla$ on $E \otimes L^{d}$ and is onto. We denote by $\nabla_{\mid x} \sigma^{\perp}$ its restriction to the orthogonal $K^{\perp}$ of $K=\operatorname{ker} \nabla_{\mid x} \sigma$,

$$
\nabla_{\mid x} \sigma^{\perp}: K^{\perp} \rightarrow \mathbb{R}\left(E \otimes L^{d}\right)_{\mid x} .
$$

Likewise, for every $\left(\dot{\sigma}_{K}, \dot{\sigma}_{\lambda}\right) \in H_{K} \oplus H_{\lambda}$, the operators $\nabla_{\mid x} \dot{\sigma}_{K}$ and $\nabla_{\mid x} \dot{\sigma}_{\lambda}$ do not depend on the choice of a connection $\nabla$ on $E \otimes L^{d}$. Finally, we write at a point $y \in \mathbb{R} X$ near $x$

$$
\sigma(y)=\sum_{i=1}^{k}\left(a_{0}^{i} \sigma_{0}^{i}+\sum_{j=1}^{n} a_{j}^{i} \sigma_{j}^{i}+\sum_{1 \leqslant l \leqslant m \leqslant n} a_{l m}^{i} \sigma_{l m}^{i}\right)(y)+o\left(|y|^{2}\right),
$$

where $\left(a_{0}^{i}\right),\left(a_{j}^{i}\right)$ and $\left(a_{l m}^{i}\right)$ are real numbers and $\left(\sigma_{0}^{i}\right),\left(\sigma_{j}^{i}\right)$ and $\left(\sigma_{l m}^{i}\right)$ are given by Definition 2.3.4. From Lemma 2.3.5 and (33), we deduce that $a_{0}^{i}=0=a_{j}^{1}$ for $1 \leqslant i \leqslant k$ and $k+1 \leqslant j \leqslant n$, and that

$$
\|\lambda\| \sqrt{\pi \delta_{L}} \sqrt{d}^{n+1}\left|a_{1}^{1}\right|=\left\|d_{\mid x} p\right\|+o(1),
$$

where the $o(1)$ term is uniformly bounded over $\mathbb{R} X$.

Lemma 3.3.1 Under the hypotheses of Theorem 3.1.3, let $(\sigma, x) \in \mathcal{I}_{i}$ and $(K, \lambda)=$ $\rho_{x}(\sigma)$. Then, $d_{\mid x} \rho_{x}^{H}$ writes

$$
\begin{aligned}
H_{K} \oplus H_{\lambda} & \rightarrow T_{K} G r\left(n-k, \operatorname{ker} d_{\mid x} p\right) \times \mathbb{R}\left(E \otimes L^{d}\right)_{\mid x}^{*} \\
\left(\dot{\sigma}_{K}, \dot{\sigma}_{\lambda}\right) & \mapsto\left(-\left(\nabla_{\mid x} \sigma^{\perp}\right)_{\mid \operatorname{ker} \lambda}^{-1} \circ \nabla_{\mid x} \dot{\sigma}_{K \mid K},-\lambda \circ \nabla_{\mid x} \dot{\sigma}_{\lambda} \circ\left(\nabla_{\mid x} \sigma^{\perp}\right)^{-1}\right) .
\end{aligned}
$$

Moreover, $\left|\operatorname{det} d_{\mid \sigma} \rho_{x}^{H}\right|^{-1}=\frac{\left|a_{1}^{1}\right|}{\|\lambda\|^{k}}\left|\operatorname{det}\left(a_{j}^{i}\right)_{2 \leqslant i, j \leqslant k}\right|^{n-k+1}(1+o(1))$, where the o(1) term is uniformly bounded over $\mathbb{R} X$.

Proof. Let $\left(\dot{\sigma}_{K}, \dot{\sigma}_{\lambda}\right) \in H_{K} \oplus H_{\lambda}$ and $\left(\sigma_{s}\right)_{s \in]-\epsilon, \epsilon[}$ be a path of $\pi_{1}\left(\pi_{2}^{-1}(x)\right)$ such that $\sigma_{0}=\sigma$ and $\dot{\sigma}_{0}=\dot{\sigma}_{K}+\dot{\sigma}_{\lambda}$. Then, for every $\left.s \in\right]-\epsilon, \epsilon\left[\right.$ and every $v_{s} \in \operatorname{ker} \nabla_{\mid x} \sigma_{s}$, there exists $\lambda_{s} \in \mathbb{R}\left(E \otimes L^{d}\right)_{\mid x}^{*}$ such that

$$
\left\{\begin{array}{l}
\nabla_{\mid x} \sigma_{s}\left(v_{s}\right)=0 \\
\lambda_{s} \circ \nabla_{\mid x} \sigma_{s}=d_{\mid x} p
\end{array} \quad\right. \text { and }
$$

By derivation, we deduce

$$
\left\{\begin{array}{l}
\nabla_{\mid x} \dot{\sigma}_{0}\left(v_{0}\right)+\nabla_{\mid x} \sigma\left(\dot{v}_{0}\right)=0 \\
\dot{\lambda}_{0} \circ \nabla_{\mid x} \sigma+\lambda \circ \nabla_{\mid x} \dot{\sigma}_{0}=0 .
\end{array}\right. \text { and }
$$

By setting $\dot{v}$ the orthogonal projection of $\dot{v}_{0}$ onto $K^{\perp}$, we deduce that

$$
\left\{\begin{array}{l}
\dot{v}=-\left(\nabla_{\mid x} \sigma^{\perp}\right)^{-1} \circ \nabla_{\mid x} \dot{\sigma}_{K}\left(v_{0}\right) \quad \text { and } \\
\dot{\lambda}_{0}=-\lambda \circ \nabla_{\mid x} \dot{\sigma}_{\lambda} \circ\left(\nabla_{\mid x} \sigma^{\perp}\right)^{-1} .
\end{array}\right.
$$


The first part of Lemma 3.3.1 follows. Now, recall that $d_{\mid x} p$ is colinear to $d x_{1}$, that $K$ is equipped with the orthonormal basis $\left(\partial / \partial x_{k+1}, \cdots, \partial / \partial x_{n}\right), K^{\perp}$ with the orthonormal basis $\left(\partial / \partial x_{1}, \cdots, \partial / \partial x_{k}\right)$, and that $\operatorname{ker} \lambda_{\mid x}$ is spanned by the orthonormal basis $\left(e_{2}, \cdots, e_{k}\right)_{\mid x}$. From Lemma 2.3.3, the map

$$
\dot{\sigma}_{K} \in H_{K} \mapsto \nabla_{\mid x} \dot{\sigma}_{K \mid K} \in L(K, \operatorname{ker} \lambda)
$$

just dilates the norm by the factor $\sqrt{\pi \delta_{L} d^{n+1}}(1+o(1))$, where the $o(1)$ term is uniformly bounded over $\mathbb{R} X$. Now, since the matrix of the restriction of $\nabla_{\mid x} \sigma^{\perp}$ to $K^{\perp} \cap \operatorname{ker} d_{\mid x} p$ in the given basis of $K^{\perp} \cap \operatorname{ker} d_{\mid x} p$ and ker $\lambda$ equals

$$
\sqrt{\pi \delta_{L} d^{n+1}}\left(a_{j}^{i}\right)_{2 \leqslant i, j \leqslant k}+o\left(\sqrt{d}^{n+1}\right),
$$

where the $o\left(\sqrt{d}^{n+1}\right)$ term is uniformly bounded over $\mathbb{R} X$. We deduce that the Jacobian determinant of the map

$$
\left.M \in L(K, \operatorname{ker} \lambda) \mapsto\left(\nabla_{\mid x} \sigma_{\mid \operatorname{ker} \lambda}^{\perp}\right)^{-1} \circ M \in L\left(K, K^{\perp} \cap \operatorname{ker} d_{\mid x} p\right)\right)
$$

equals

$$
\left(\left(\sqrt{\pi \delta_{L} d^{n+1}}\right)^{k-1}\left|\operatorname{det}\left(a_{j}^{i}\right)_{2 \leqslant i, j \leqslant k}\right|(1+o(1))\right)^{k-n} .
$$

The Jacobian determinant of the map

$$
\dot{\sigma}_{K} \in H_{K} \mapsto\left(\nabla_{\mid x} \sigma^{\perp}\right)_{\mid \text {ker } \lambda}^{-1} \circ \nabla_{\mid x} \dot{\sigma}_{K \mid K} \in T_{K} \operatorname{Gr}\left(n-k, \operatorname{ker} d_{\mid x} p\right)
$$

thus equals $\left|\operatorname{det}\left(a_{j}^{i}\right)_{2 \leqslant i, j \leqslant k}\right|^{k-n}+o(1)$, where the $o(1)$ is uniformly bounded over $\mathbb{R} X$. Likewise, from Lemma 2.3.3, the map

$$
\dot{\sigma}_{\lambda} \in H_{\lambda} \mapsto \lambda \circ \nabla_{\mid x} \dot{\sigma}_{\lambda} \in\left(K^{\perp}\right)^{*}
$$

just dilates the norm by a factor $\sqrt{\pi \delta_{L} d^{n+1}}\|\lambda\|+o\left(\sqrt{d}^{n+1}\right)$, where the $o\left(\sqrt{d}^{n+1}\right)$ is uniformly bounded over $\mathbb{R} X$, while the Jacobian determinant of the map

$$
M \in\left(K^{\perp}\right)^{*} \mapsto M \circ\left(\nabla_{\mid x} \sigma^{\perp}\right)^{-1} \in \mathbb{R}\left(E \otimes L^{d}\right)_{\mid x}^{*}
$$

equals $\left(\sqrt{\pi \delta_{L}} \sqrt{d}^{n+1}\right)^{-k}\left|\operatorname{det}\left(a_{j}^{i}\right)_{1 \leqslant i, j \leqslant k}\right|^{-1}(1+o(1))$ so that the Jacobian determinant of the map

$$
\dot{\sigma}_{\lambda} \in H_{\lambda} \mapsto \lambda \circ \nabla_{\mid x} \dot{\sigma}_{\lambda} \circ\left(\nabla_{\mid x} \sigma^{\perp}\right)^{-1} \in \mathbb{R}\left(E \otimes L^{d}\right)_{\mid x}^{*}
$$

equals $\|\lambda\|^{k}\left|\operatorname{det}\left(a_{j}^{i}\right)_{1 \leqslant i, j \leqslant k}\right|^{-1}+o(1)$, with a $o(1)$ uniformly bounded over $\mathbb{R} X$. As a consequence,

$$
\left|\operatorname{det} d_{\mid \sigma} \rho_{x}^{H}\right|^{-1}=\|\lambda\|^{-k}\left|\operatorname{det}\left(a_{j}^{i}\right)_{2 \leqslant i, j \leqslant k}\right|^{n-k+1}\left|a_{1}^{1}\right|(1+o(1)),
$$

with a $o(1)$ uniformly bounded over $\mathbb{R} X$, since the relation $\lambda \circ \nabla_{\mid x} \sigma=d_{\mid x} p$ implies that $a_{j}^{1}$ vanishes for $2 \leqslant j \leqslant n$. 


\subsubsection{Jacobian determinant of the evaluation map}

Again, under the hypotheses of Theorem 3.1 .3 and for $(\sigma, x) \in \mathcal{I}_{i}$, we set for every $y$ in a neighbourhood of $x$,

$$
\sigma(y)=\sum_{i=1}^{k}\left(a_{0}^{i} \sigma_{0}^{i}+\sum_{j=1}^{n} a_{j}^{i} \sigma_{j}^{i}+\sum_{1 \leqslant l \leqslant m \leqslant n} a_{l m}^{i} \sigma_{l m}^{i}\right)(y)+o\left(|y|^{2}\right),
$$

where $a_{0}^{i}, a_{j}^{i}$ and $a_{l m}^{i}$ are real numbers. We then set, for $1 \leqslant l, m \leqslant n, \tilde{a}_{l l}^{1}=\sqrt{2} a_{l l}^{1}$, $\tilde{a}_{l m}^{1}=a_{l m}^{1}$ if $l<m$ and $\tilde{a}_{l m}^{1}=a_{m l}^{1}$ if $l>m$. We denote by $d_{\mid \sigma} e v_{(\sigma, x)}^{H}$ the restriction of $d_{\mid \sigma} e v_{(\sigma, x)}$ to $H_{x}$, see (37) and by $\operatorname{det} d_{\mid \sigma} e v_{(\sigma, x)}^{H}$ its Jacobian determinant computed in the given basis of $H_{x}$ and orthonormal basis of $T_{x} \mathbb{R} X$.

Lemma 3.3.2 Under the hypotheses of Theorem 3.1.3, let $(\sigma, x) \in \mathcal{I}_{i}$. Then,

$$
\left|\operatorname{det} d_{\mid \sigma} e v_{(\sigma, x)}^{H}\right|^{-1}=\sqrt{\pi^{n} d^{n}}\left|a_{1}^{1}\right|\left|\operatorname{det}\left(a_{j}^{i}\right)_{2 \leqslant i, j \leqslant k}\right|\left|\operatorname{det}\left(\tilde{a}_{l m}^{1}\right)_{k+1 \leqslant l, m \leqslant n}\right|(1+o(1)),
$$

where the o(1) term has poles of order at most $n-k$ near the critical points of $p$.

Remark 3.3.3 In Lemma 3.3.2, a function $f$ is said to have a pole of order at most $n-k$ near a point $x$ if $r^{n-k} f$ is bounded near $x$, where $r$ denotes the distance function to $x$. Such a function thus belongs to $L^{1}\left(\mathbb{R} X, d v o l_{h}\right)$.

Proof. We choose a torsion free connection $\nabla^{T X}$ (resp. a connection $\nabla^{E \otimes L^{d}}$ ) on $\mathbb{R} X \backslash \operatorname{Crit}(p)$ (resp. on $E \otimes L^{d}$ ) such that $\nabla^{T X} d p=0$. They induce a connection on $T^{*} X \otimes E \otimes L^{d}$ which makes it possible to differentiate twice the elements of $\mathbb{R} H^{0}\left(X, E \otimes L^{d}\right)$. The tangent space of $\mathcal{I}_{i}$ then reads

$$
\begin{aligned}
T_{(\sigma, x)} \mathcal{I}_{i}= & \left\{(\dot{\sigma}, \dot{x}) \in \mathbb{R} H^{0}\left(X, E \otimes L^{d}\right) \times T_{x} \mathbb{R} X \mid \dot{\sigma}(x)+\nabla_{\dot{x}} \sigma=0\right. \text { and } \\
& \left.\exists \dot{\lambda} \in \mathbb{R}\left(E \otimes L^{d}\right)_{\mid x}^{*}, \dot{\lambda} \circ \nabla_{\mid x} \sigma+\lambda \circ \nabla_{\mid x} \dot{\sigma}+\lambda \circ \nabla_{\dot{x}, .}^{2} \sigma=0\right\} .
\end{aligned}
$$

Recall that $T_{x} \mathbb{R} X$ is the direct sum $K \oplus K^{\perp}$, where $K=\operatorname{ker} \nabla_{\mid x} \sigma$. We write $\dot{x}=$ $\left(\dot{x}_{K}, \dot{x}_{K^{\perp}}\right)$ the coordinates of $\dot{x}$ in this decomposition. From the first equation we deduce, keeping the notations of $\$ 3.3 .1$, that $\dot{x}_{K^{\perp}}=-\left(\nabla_{\mid x} \sigma^{\perp}\right)^{-1}(\dot{\sigma}(x))$. From Lemma 2.3.3, the evaluation map at $x$

$$
\dot{\sigma} \in\left\langle\left(\sigma_{0}^{i}\right)_{1 \leqslant i \leqslant k}\right\rangle \mapsto \dot{\sigma}(x) \in E \otimes L_{\mid x}^{d}
$$

just dilates the norm by a factor $\sqrt{\delta_{L} d^{n}}(1+o(1))$, where the $o(1)$ term is uniformly bounded over $\mathbb{R} X$, while

$$
\left|\operatorname{det}\left(\nabla_{\mid x} \sigma^{\perp}\right)\right|=\left(\sqrt{\pi \delta_{L} d^{n+1}}\right)^{k}\left|\operatorname{det}\left(a_{j}^{i}\right)_{1 \leqslant i, j \leqslant k}\right|(1+o(1)) .
$$

We deduce by composition that the Jacobian of the map

$$
\dot{\sigma} \in\left\langle\left(\sigma_{0}^{i}\right)_{1 \leqslant i \leqslant k}\right\rangle \mapsto \dot{x}_{K^{\perp}}=-\left(\nabla_{\mid x} \sigma^{\perp}\right)^{-1}(\dot{\sigma}(x))
$$

equals $\left(\sqrt{\pi^{k} d^{k}}\left|\operatorname{det}\left(a_{j}^{i}\right)_{2 \leqslant i, j \leqslant k}\right|\left|a_{1}^{1}\right|\right)^{-1}(1+o(1))$, where the $o(1)$ term is uniformly bounded over $\mathbb{R} X$. Now, equation (43) restricted to $K$ reads

$$
\lambda \circ \nabla_{\dot{x}_{K}, .}^{2} \sigma_{\mid K}=-\lambda \circ \nabla_{\mid x} \dot{\sigma}_{\mid K} .
$$


From Lemma 2.3.3, the map

$$
\dot{\sigma} \in\left\langle\left(\sigma_{j}^{1}\right)_{k+1 \leqslant j \leqslant n}\right\rangle \mapsto-\lambda \circ \nabla_{\mid x} \dot{\sigma}_{\mid K} \in K^{*}
$$

just dilates the norm by a factor $\|\lambda\| \sqrt{\pi \delta_{L} d^{n+1}}(1+o(1))$, with $o(1)$ term is uniformly bounded over $\mathbb{R} X$. Likewise, from Lemma 2.3.3, the Jacobian of the map $\lambda \circ \nabla^{2} \sigma_{\mid K}$ : $K \rightarrow K^{*}$ equals

$$
\left(\|\lambda\| \pi \sqrt{\delta_{L} d^{n+2}}\right)^{n-k}\left|\operatorname{det}\left(\tilde{a}_{l m}^{1}\right)_{k+1 \leqslant l, m \leqslant n}\right|(1+o(1)) .
$$

Here, the $o(1)$ term is no more uniformly bounded over $\mathbb{R} X$ though. Indeed, from Lemma 2.3.5 and (41),

$$
\lambda \circ \nabla^{2} \sigma_{\mid K}=a_{1}^{1}\left(\|\lambda\| \sqrt{\pi \delta_{L} d^{n+1}}\right)\left(\nabla^{T X} d x_{1}\right)+\sum_{1 \leqslant l \leqslant m \leqslant n} \tilde{a}_{l m}^{1}\left(\|\lambda\| \sqrt{\pi \delta_{L} d^{n+2}}\right) d x_{l} \otimes d x_{m},
$$

since the relation $\lambda \circ \nabla_{\mid x} \sigma=d_{\mid x} p$ imposes that $a_{j}^{1}$ vanishes for $j>1$. Moreover, since $d p=\sum_{i=1}^{n} \alpha_{i} d x_{i}$, with $\alpha_{2}(x)=\cdots=\alpha_{n}(x)=0$ and $\left|\alpha_{1}(x)\right|=\left\|d_{\mid x} p\right\|$, we get that

$$
0=\nabla^{T X}(d p)_{\mid K}=\alpha_{1}\left(\nabla^{T X} d x_{1}\right)_{\mid K}+\sum_{i=1}^{n}\left(d \alpha_{i} \otimes d x_{i}\right)_{\mid K}
$$

so that $\left\|\nabla^{T X} d x_{1 \mid K}\right\|=\frac{1}{\left\|d_{\mid x} p\right\|}\left\|\sum_{i=1}^{n} d \alpha_{i} \otimes d x_{i}\right\|$ has a pole of order one at $x$. In formula (44), the $o(1)$ term has thus a pole of order at most $n-k$ near the critical points of $p$.

We deduce that the Jacobian determinant of the map

$$
\dot{\sigma} \in\left\langle\left(\sigma_{j}^{1}\right)_{k+1 \leqslant j \leqslant n}\right\rangle \mapsto \dot{x}_{K}=-\left(\lambda \circ \nabla^{2} \sigma_{\mid K}\right)^{-1} \circ\left(\lambda \circ \nabla_{\mid x} \dot{\sigma}_{\mid K}\right) \in K
$$

equals $\left(\sqrt{\pi^{n-k} d^{n-k}}\left|\operatorname{det}\left(\tilde{a}_{l m}^{1}\right)_{k+1 \leqslant l, m \leqslant n}\right|\right)^{-1}(1+o(1))$, up to sign, where $o(1)$ term has a pole of order at most $n-k$ near the critical points of $p$. The result follows.

\subsection{Proof of Theorem 3.1 .3}

\subsubsection{The case $k<n$}

From Proposition 2.4.1 we know that

$$
\mathbb{E}\left(\nu_{i}\right)=\frac{1}{\sqrt{\pi^{n} d^{n}}}\left(\int_{\pi_{1}\left(\pi_{2}^{-1}(x)\right)}\left|\operatorname{det} d_{\mid \sigma} e v_{(\sigma, x)}^{\perp}\right|^{-1} d \mu_{\mathbb{R}}(\sigma)\right) d v o l_{h_{L}}
$$

From the coarea formula (see [10]), we likewise deduce that

$$
\begin{aligned}
\mathbb{E}\left(\nu_{i}\right)= & \frac{1}{\sqrt{\pi^{n} d^{n}}}\left(\int_{\operatorname{Gr}\left(n-k, \operatorname{ker} d_{\mid x} p\right) \times \mathbb{R}\left(E \otimes L^{d}\right)_{\mid x}^{*} \backslash\{0\}} e^{-\left(a_{1}^{1}\right)^{2}} \frac{d K \wedge d \lambda}{\sqrt{\pi}} \ldots\right. \\
& \left.\cdots \int_{\rho_{x}^{-1}(K, \lambda)}\left|\operatorname{det} d_{\mid \sigma} e v_{(\sigma, x)}^{\perp}\right|^{-1}\left|\operatorname{det} d_{\mid \sigma} \rho_{x}^{\perp}\right|^{-1} d \mu_{\mathbb{R}}(\sigma)\right) d v o l_{h_{L}},
\end{aligned}
$$

since with the notations (41), $\sigma \in \rho_{x}^{-1}(K, \lambda)$ if and only if $\forall i \in\{1, \cdots, k\}$ and $\forall j \in$ $\{k+1, \cdots, n\}, a_{0}^{i}=0=a_{j}^{i}$ while $\forall j \geqslant 2, a_{j}^{1}=0$ and $\left|a_{1}^{1}\right|=\frac{\left\|d_{\mid x} p\right\|}{\|\lambda\| \sqrt{\pi \delta_{L}} \sqrt{d}^{n+1}}$. From 
Lemma 2.3.6 and the relation (40), we deduce that for every $x \in \mathbb{R} X \backslash \operatorname{Crit}(p)$ and every $(K, \lambda) \in \operatorname{Gr}\left(n-k\right.$, ker $\left.d_{\mid x} p\right) \times \mathbb{R}\left(E \otimes L^{d}\right)_{\mid x}^{*} \backslash\{0\}$,

$$
\begin{aligned}
& \int_{\rho_{x}^{-1}(K, \lambda)}\left|\operatorname{det} d_{\mid \sigma} e v_{(\sigma, x)}^{\perp}\right|^{-1}\left|\operatorname{det} d_{\mid \sigma} \rho_{x}^{\perp}\right|^{-1} d \mu_{\mathbb{R}}(\sigma) \\
\underset{d \rightarrow \infty}{\sim} & \int_{\rho_{x}^{-1}(K, \lambda)}\left|\operatorname{det} d_{\mid \sigma} e v_{(\sigma, x)}^{H}\right|^{-1}\left|\operatorname{det} d_{\mid \sigma} \rho_{x}^{H}\right|^{-1} d \mu_{\mathbb{R}}(\sigma) .
\end{aligned}
$$

Thus, from Lemmas 3.3.1, 3.3.2 and 3.2.2, $\mathbb{E}\left(\nu_{i}\right)$ converges to

$$
\begin{aligned}
& \int_{M_{k-1}(\mathbb{R})}\left|\operatorname{det}\left(a_{j}^{i}\right)_{2 \leqslant i, j \leqslant k}\right|^{n-k+2} d \mu\left(a_{j}^{i}\right) \int_{S y m_{\mathbb{R}}(i, n-k-i)}\left|\operatorname{det}\left(\tilde{a}_{l m}^{1}\right)_{k+1 \leqslant l, m \leqslant n}\right| d \mu\left(\tilde{a}_{l m}^{1}\right) \ldots \\
& \ldots \int_{\operatorname{Gr}\left(n-k, \operatorname{ker} d_{\mid x} p\right) \times \mathbb{R}\left(E \otimes L^{d}\right)_{\mid x}^{*} \backslash\{0\}} \frac{\left(a_{1}^{1}\right)^{2} e^{-\left(a_{1}^{1}\right)^{2}}}{\|\lambda\|^{k}} \frac{d K \wedge d \lambda}{\sqrt{\pi}^{(n-k)(k-1)+k}},
\end{aligned}
$$

where the convergence is dominated by a function in $L^{1}\left(\mathbb{R} X, d v o l_{h_{L}}\right)$, see Remark 3.3.3. We deduce that $\mathbb{E}\left(\nu_{i}\right)$ gets equivalent to

$$
\frac{\left\|d_{\mid x} p\right\|^{2}}{\delta_{L} d^{n+1} \sqrt{\pi}^{k+2}} V_{k-1, n-1} E_{k-1}\left(|\operatorname{det}|^{n-k+2}\right) e_{\mathbb{R}}(i, n-k-i)\left(\int_{\mathbb{R}\left(E \otimes L^{d}\right)_{\mid x}^{*} \backslash\{0\}} \frac{e^{-\left(a_{1}^{1}\right)^{2}}}{\|\lambda\|^{k+2}} d \lambda\right) d v o l_{h_{L}} .
$$

Now,

$$
\begin{aligned}
\frac{\left\|d_{\mid x} p\right\|^{2}}{\pi \delta_{L} d^{n+1}} \int_{\mathbb{R}\left(E \otimes L^{d}\right)_{\mid x}^{*} \backslash\{0\}} \frac{e^{-\left(a_{1}^{1}\right)^{2}}}{\|\lambda\|^{k+2}} d \lambda & =\frac{\operatorname{Vol}\left(S^{k-1}\right)\left\|d_{\mid x} p\right\|^{2}}{\pi \delta_{L} d^{n+1}} \int_{0}^{+\infty} \frac{e^{-\left(a_{1}^{1}\right)^{2}}}{\|\lambda\|^{3}} d\|\lambda\| \\
& =\operatorname{Vol}\left(S^{k-1}\right) \int_{0}^{+\infty} e^{-r^{2}} r d r=\frac{1}{2} \operatorname{Vol}\left(S^{k-1}\right) .
\end{aligned}
$$

Since $\operatorname{Vol}\left(S^{k-1}\right)=\frac{2 \sqrt{\pi}^{k}}{\Gamma(k / 2)}$, we finally deduce that $\mathbb{E}\left(\nu_{i}\right)$ weakly converges to

$$
\frac{1}{\Gamma(k / 2)} V_{k-1, n-1} E_{k-1}\left(|\operatorname{det}|^{n-k+2}\right) e_{\mathbb{R}}(i, n-k-i) d v o l_{h_{L}},
$$

where the convergence is dominated by a function in $L^{1}\left(\mathbb{R} X, d v \operatorname{lol}_{h_{L}}\right)$.

\subsubsection{The case $k=n$}

When the rank of $E$ equals the dimension of $X$, the vanishing locus of a generic section $\sigma$ of $\mathbb{R} H^{0}\left(X, E \otimes L^{d}\right)$ is a finite set of points. We set $\nu=\frac{1}{\sqrt{d}^{n}} \sum_{x \in \mathbb{R} C_{\sigma}} \delta_{x}$, and define the incidence variety as

$$
\mathcal{I}=\left\{(\sigma, x) \in\left(\mathbb{R} H^{0}\left(X, E \otimes L^{d}\right) \backslash \mathbb{R} \Delta_{d}\right) \times \mathbb{R} X \mid \sigma(x)=0\right\} .
$$

The projections $\pi_{1}$ and $\pi_{2}$ are defined by (31) and (32). As before, for every $\left(\sigma_{0}, x_{0}\right) \in$ $\left(\mathbb{R} H^{0}\left(X, E \otimes L^{d}\right) \backslash \mathbb{R} \Delta_{d}\right) \times \mathbb{R} X, \pi_{1}$ is invertible in a neighbourhood $\mathbb{R} U$ of $\sigma_{0}$, defining an evaluation map at the critical point

$$
e v_{\left(\sigma_{0}, x_{0}\right)}: \sigma \in \mathbb{R} U \mapsto \pi_{2} \circ \pi_{1}^{-1}(\sigma)=x \in \mathbb{R} C_{\sigma} \cap \mathbb{R} V
$$


where $\mathbb{R} V$ denotes a neighbourhood of $x_{0}$ in $\mathbb{R} X$, compare $\S 2.4 .2$ of [14. We denote by $d_{\mid \sigma_{0}} e v_{\left(\sigma_{0}, x_{0}\right)}^{\perp}$ the restriction of its differential map $d_{\mid \sigma_{0}} e v_{\left(\sigma_{0}, x_{0}\right)}$ at $\sigma_{0}$ to the orthogonal complement of $\pi_{1}\left(\pi_{2}^{-1}\left(x_{0}\right)\right)$ in $\mathbb{R} H^{0}\left(X, E \otimes L^{d}\right)$. Then, from Proposition 3.2.1,

$$
\mathbb{E}(\nu)=\frac{1}{\sqrt{d}^{n}}\left(\pi_{2}\right)_{*}\left(\pi_{1}^{*} d \mu_{\mathbb{R}}\right)_{\mid x}=\frac{1}{\sqrt{\pi d}^{n}} \int_{\pi_{1}\left(\pi_{2}^{-1}(x)\right)}\left|\operatorname{det} d_{\mid \sigma} e v_{(\sigma, x)}^{\perp}\right|^{-1} d \mu_{\mathbb{R}}(\sigma) d v o l_{h_{L}} .
$$

The space $H_{x}=\left\langle\left(\sigma_{0}^{i}\right)_{1 \leqslant i \leqslant k}\right\rangle$ is a complement to $T_{\sigma} \pi_{1}\left(\pi_{2}^{-1}(x)\right)$ in $\mathbb{R} H^{0}\left(X, E \otimes L^{d}\right)$ and in the decomposition (41), $a_{0}^{i}=0$ for every $i=1, \cdots, k$. The tangent space of $\mathcal{I}$ at $(\sigma, x)$ reads

$$
T_{(\sigma, x)} \mathcal{I}=\left\{(\dot{\sigma}, \dot{x}) \in \mathbb{R} H^{0}\left(X, E \otimes L^{d}\right) \times T_{x} \mathbb{R} X \mid \dot{\sigma}(x)+\nabla_{\mid x} \sigma(\dot{x})=0\right\} .
$$

As in the proof of Lemma 3.3.2, we deduce that the Jacobian determinant of the map

$$
\dot{\sigma} \in H_{x} \mapsto \dot{x}=-\left(\nabla_{\mid x} \sigma^{\perp}\right)^{-1}(\dot{\sigma}(x)) \in T_{x} \mathbb{R} X
$$

equals $\sqrt{\pi^{n} d^{n}}\left|\operatorname{det}\left(a_{j}^{i}\right)_{1 \leqslant i, j \leqslant n}\right|(1+o(1))$, so that

$$
\left|\operatorname{det} d_{\mid \sigma} e v_{(\sigma, x)}^{H}\right|^{-1}=\sqrt{\pi d^{n}}\left|\operatorname{det}\left(a_{j}^{i}\right)_{1 \leqslant i, j \leqslant n}\right|(1+o(1)),
$$

where the $o(1)$ term is uniformly bounded over $\mathbb{R} X$. From lemma 2.3.6 we deduce that $\mathbb{E}(\nu)$ gets equivalent to

$$
\left(\int_{M_{n}(\mathbb{R})}\left|\operatorname{det}\left(a_{j}^{i}\right)_{1 \leqslant i, j \leqslant n}\right| d \mu\left(a_{j}^{i}\right)\right) \operatorname{dvol}_{h_{L}}=E_{n}(|\operatorname{det}|) d v o l_{h_{L}} .
$$

Formula (15.4.12) of [23], see Remark 3.1.1, now gives

$$
E_{n}(|\operatorname{det}|)=\frac{\Gamma\left(\frac{n+1}{2}\right)}{\Gamma(1 / 2)}=\frac{1}{\operatorname{Vol}_{F S}\left(\mathbb{R} P^{n}\right)},
$$

see Remark 2.14 of [14], hence the result.

\subsection{Equidistribution of critical points in the complex case}

Let $X$ be a smooth complex projective manifold of dimension $n,\left(L, h_{L}\right)$ be a holomorphic Hermitian line bundle of positive curvature $\omega$ over $X$ and $\left(E, h_{E}\right)$ be a rank $k$ holomorphic Hermitian vector bundle, with $1 \leqslant k \leqslant n$. For every $d>0$, we denote by $L^{d}$ the $d$ th tensor power of $L$ and by $h^{d}$ the induced Hermitian metric on $L^{d}$. We denote by $H^{0}\left(X, L^{d}\right)$ its complex vector space of global holomorphic sections and by $N_{d}$ the dimension of $H^{0}\left(X, L^{d}\right)$. We denote then by $\langle.,$.$\rangle the L^{2}$-Hermitian product on this vector space, defined by the relation

$$
\forall \sigma, \tau \in H^{0}\left(X, L^{d}\right),\langle\sigma, \tau\rangle=\int_{X} h^{d}(\sigma, \tau) d x .
$$

The associated Gaussian measure is denoted by $\mu_{\mathbb{C}}$. It is defined, for every open subset $U$ of $H^{0}\left(X, L^{d}\right)$, by

$$
\mu_{\mathbb{C}}(U)=\frac{1}{\pi^{N_{d}}} \int_{U} e^{-\|\sigma\|^{2}} d \sigma
$$


where $d \sigma$ denotes the Lebesgue measure of $H^{0}\left(X, L^{d}\right)$. For every $d>0$, we denote by $\Delta^{d}$ the discriminant hypersurface of $H^{0}\left(X, E \otimes L^{d}\right)$, that is the set of sections $\sigma \in H^{0}\left(X, E \otimes L^{d}\right)$ which do not vanish transversally. For every $\sigma \in H^{0}\left(X, E \otimes L^{d}\right) \backslash\{0\}$, we denote by $C_{\sigma}$ the vanishing locus of $\sigma$ in $X$. For every $\sigma \in H^{0}\left(X, E \otimes L^{d}\right) \backslash \Delta^{d}$, $C_{\sigma}$ is then a smooth codimension $k$ complex submanifold of $X$. We equip $X$ with a Lefschetz pencil $p: X \rightarrow \mathbb{C} P^{1}$. We then denote, for every $d>0$, by $\Delta_{p}^{d}$ the set of sections $\left.\sigma \in H^{0}\left(X, E \otimes L^{d}\right)\right)$ such that $\sigma \in \Delta^{d}$, or $C_{\sigma}$ intersects the critical locus of $p$, or the restriction of $p$ to $C_{\sigma}$ is not a Lefschetz pencil. For $d$ large enough, this extended discriminant locus is of measure 0 for the measure $\mu_{\mathbb{C}}$.

For every $\sigma \in H^{0}\left(X, E \otimes L^{d}\right) \backslash \Delta_{p}^{d}$, we denote by $\operatorname{Crit}\left(p_{\mid C_{\sigma}}\right)$ the set of critical points of the restriction of $p$ to $C_{\sigma}$ and set, for $1 \leqslant k \leqslant n-1$,

$$
\nu\left(C_{\sigma}\right)=\frac{1}{d^{n}} \sum_{x \in \operatorname{Crit}\left(p_{\mid C_{\sigma}}\right)} \delta_{x}
$$

where $\delta_{x}$ denotes the Dirac measure of $X$ at the point $x$. When $k=n, \nu\left(C_{\sigma}\right)=$ $\frac{1}{d^{n}} \sum_{x \in C_{\sigma}} \delta_{x}$.

Theorem 3.5.1 Let $X$ be a smooth complex projective manifold of dimension $n$, $\left(L, h_{L}\right)$ be a holomorphic Hermitian line bundle of positive curvature $\omega$ over $X$ and $\left(E, h_{E}\right)$ be a rank $k$ holomorphic Hermitian vector bundle, with $1 \leqslant k \leqslant n$. Let $p: X \rightarrow \mathbb{C} P^{1}$ be a Lefschetz pencil. Then, the measure $\mathbb{E}(\nu)$ defined by (47) weakly converges to $\left(\begin{array}{l}n-1 \\ k-1\end{array}\right) \omega^{n}$ as d grows to infinity.

When $k=1$, Theorem 3.5.1 reduces to Theorem 3 of [15], see also Theorem 1.3 of [14.

Proof. The proof goes along the same lines as the one of Theorem 3.1.2, so we only give a sketch of it. Firstly, the analogue of Proposition 3.2.1 provides

$$
\mathbb{E}(\nu)=\frac{1}{d^{n}}\left(\pi_{2}\right)_{*}\left(\pi_{1}^{*} d \mu_{\mathbb{C}}\right),
$$

and at every point $x \in X \backslash(\operatorname{Crit}(p) \cup \operatorname{Base}(p))$, where $\operatorname{Base}(p)$ denotes the base locus of $p$,

$$
\left(\pi_{2}\right)_{*}\left(\pi_{1}^{*} d \mu_{\mathbb{C}}\right)_{\mid x}=\frac{1}{\pi^{n}} \int_{\pi_{1}\left(\pi_{2}^{-1}(x)\right)}\left|\operatorname{det} d_{\mid \sigma} e v_{(\sigma, x)}^{\perp}\right|^{-2} d \mu_{\mathbb{R}}(\sigma) \frac{\omega^{n}}{n !},
$$

see Proposition 2.10 of [14]. Choosing complex coefficients in decomposition (41), Lemmas 3.3.1 and 3.3.2 remain valid in the complex setting, see Remark 2.3.8. We deduce that

$$
\begin{aligned}
\mathbb{E}(\nu)= & \frac{1}{\pi^{n} d^{n}}\left(\int_{\pi_{1}\left(\pi_{2}^{-1}(x)\right)}\left|\operatorname{det} d_{\mid \sigma} e v_{(\sigma, x)}^{\perp}\right|^{-2} d \mu_{\mathbb{C}}(\sigma)\right) \frac{\omega^{n}}{n !} \\
\underset{d \rightarrow \infty}{\sim} & \frac{1}{\pi^{n} d^{n}}\left(\int_{\operatorname{Gr}_{\mathbb{C}}\left(n-k, \operatorname{ker} d_{\mid x} p\right) \times\left(E \otimes L^{d}\right)_{\mid x}^{*} \backslash\{0\}} e^{-\left|a_{1}^{1}\right|^{2}} \frac{d K \wedge d \lambda}{\pi^{(n-k)(k-1)+k} \ldots}\right. \\
& \left.\ldots \int_{\rho_{x}^{-1}(K, \lambda)}\left|\operatorname{det} d_{\mid \sigma} e v_{(\sigma, x)}^{\perp}\right|^{-2}\left|\operatorname{det} d_{\mid \sigma} \rho_{x}^{\perp}\right|^{-2} d \mu_{\mathbb{C}}(\sigma)\right) \frac{\omega^{n}}{n !},
\end{aligned}
$$

with $\left|a_{1}^{1}\right|$ given by (40), see Lemma 2.3.6 as before. Here, $\operatorname{Gr}_{\mathbb{C}}\left(n-k\right.$, ker $\left.d_{\mid x} p\right)$ denotes the Grassmann manifold of $n-k$-dimensional complex linear subspaces of ker $d_{\mid x} p$. 
From the complex versions of Lemma 2.3.5 and 2.3.6, see Remark 2.3.8 and the relation (40), we deduce that for every $x \in X \backslash(\operatorname{Crit}(p) \cup \operatorname{Base}(p))$ and every $(K, \lambda) \in$ $\operatorname{Gr}\left(n-k, \operatorname{ker} d_{\mid x} p\right) \times\left(E \otimes L^{d}\right)_{\mid x}^{*} \backslash\{0\}$,

$$
\begin{aligned}
& \int_{\rho_{x}^{-1}(K, \lambda)}\left|\operatorname{det} d_{\mid \sigma} e v_{(\sigma, x)}^{\perp}\right|^{-2}\left|\operatorname{det} d_{\mid \sigma} \rho_{x}^{\perp}\right|^{-2} d \mu_{\mathbb{C}}(\sigma) \\
\underset{d \rightarrow \infty}{\sim} & \frac{\left|a_{1}^{1}\right|^{4} \pi^{n} d^{n}}{\|\lambda\|^{2 k}} \int_{M_{k-1}(\mathbb{C})}\left|\operatorname{det}\left(a_{j}^{i}\right)_{2 \leqslant i, j \leqslant k}\right|^{2(n-k+2)} d \mu\left(a_{j}^{i}\right)_{\ldots} \ldots \\
& \ldots \int_{\text {Sym }_{\mathbb{C}}(n-k)}\left|\operatorname{det}\left(\tilde{a}_{l m}^{1}\right)_{k+1 \leqslant l, m \leqslant n}\right|^{2} d \mu\left(\tilde{a}_{l m}^{1}\right) .
\end{aligned}
$$

We deduce that $\mathbb{E}(\nu)$ is equivalent to

$$
\begin{aligned}
& \frac{\left\|d_{\mid x} p\right\|^{4}}{\left(\pi \delta_{L} d^{n+1}\right)^{2}} \frac{1}{\pi^{(n-k)(k-1)+k}} \operatorname{Vol}\left(\operatorname{Gr}_{\mathbb{C}}(k-1, n-1)\right) \ldots \\
& \ldots E_{k-1}^{\mathbb{C}}\left(|\operatorname{det}|^{2(n-k+2)}\right) e_{\mathbb{C}}(n-k)\left(\int_{\left(E \otimes L^{d}\right)_{\mid x}^{*} \backslash\{0\}} \frac{e^{-\left|a_{1}^{1}\right|^{2}}}{\|\lambda\|^{2(k+2)}} d \lambda\right) \frac{\omega^{n}}{n !},
\end{aligned}
$$

where $e_{\mathbb{C}}(n-k)=\int_{S y m_{\mathbb{C}}(n-k)}|\operatorname{det} A|^{2} d \mu_{\mathbb{C}}(A)$ and

$$
E_{k-1}^{\mathbb{C}}\left(|\operatorname{det}|^{2(n-k+2)}\right)=\int_{M_{k-1}(\mathbb{C})}|\operatorname{det} A|^{2(n-k+2)} d \mu_{\mathbb{C}}(A) .
$$

Now,

$$
\begin{aligned}
\frac{\left\|d_{\mid x} p\right\|^{4}}{\left(\pi \delta_{L} d^{n+1}\right)^{2}} \int_{\left(E \otimes L^{d}\right)_{\mid x}^{*} \backslash\{0\}} \frac{e^{-\left|a_{1}^{1}\right|^{2}}}{\|\lambda\|^{2 k+4}} d \lambda & =\operatorname{Vol}\left(S^{2 k-1}\right) \frac{\left\|d_{\mid x} p\right\|^{4}}{\left(\pi \delta_{L} d^{n+1}\right)^{2}} \int_{0}^{+\infty} \frac{e^{-\left|a_{1}^{1}\right|^{2}}}{\|\lambda\|^{5}} d\|\lambda\| \\
& =\operatorname{Vol}\left(S^{2 k-1}\right) \int_{0}^{+\infty} e^{-r^{2}} r^{3} d r=\frac{1}{2} \operatorname{Vol}\left(S^{2 k-1}\right) .
\end{aligned}
$$

Hence, $\mathbb{E}(\nu)$ is equivalent to

$$
\frac{1}{2 \pi^{(n-k)(k-1)+k}} \operatorname{Vol}\left(\operatorname{Gr}_{\mathbb{C}}(k-1, n-1)\right) \operatorname{Vol}\left(S^{2 k-1}\right) E_{k-1}^{\mathbb{C}}\left(|\operatorname{det}|^{2(n-k+2)}\right) e_{\mathbb{C}}(n-k) \frac{\omega^{n}}{n !},
$$

where $e_{\mathbb{C}}(n-k)=(n-k+1)$ ! by Proposition 3.8 of [14], $\operatorname{Vol}\left(S^{2 k-1}\right)=2 \pi^{k} /(k-1)$ !,

$$
E_{k-1}^{\mathbb{C}}\left(|\operatorname{det}|^{2(n-k+2)}\right)=\frac{\prod_{j=1}^{k-1} \Gamma((n-k+2)+j)}{\prod_{j=1}^{k-1} \Gamma(j)}=\frac{\prod_{j=n-k+3}^{n+1} \Gamma(j)}{\prod_{j=1}^{k-1} \Gamma(j)}
$$

by formula 15.4 .12 of [23] and

$$
\operatorname{Vol}\left(\operatorname{Gr}_{\mathbb{C}}(k-1, n-1)\right)=\frac{\prod_{j=1}^{k-1} \Gamma(j)}{\prod_{j=n-k+1}^{n-1} \Gamma(j)} \pi^{(k-1)(n-k)}
$$

by a computation analogous to the one given in the real case by Remark 3.1.1. We conclude that $\mathbb{E}(\nu)$ weakly converges to $\left(\begin{array}{l}k-1 \\ n-1\end{array}\right) \omega^{n}$, where the convergence is dominated by a function in $L^{1}\left(X, \frac{\omega^{n}}{n !}\right)$, for it has poles of order at most $2(n-k)$ near the critical points of $p$ and at most 2 near the base points, see [15]. 
Corollary 3.5.2 Under the hypotheses of Theorem 3.5.1, for every generic $\sigma \in$ $\mathbb{R} H^{0}\left(X, E \otimes L^{d}\right)$, let $\mid$ Crit $p_{\left|C_{\sigma}\right|} \mid$ be the number of critical points of $p_{\mid C_{\sigma}}$. Then,

$$
\frac{1}{d^{n}} \mathbb{E}\left(\mid \text { Crit } p_{\left|C_{\sigma}\right|} \mid\right) \underset{d \rightarrow \infty}{\sim}\left(\begin{array}{l}
k-1 \\
n-1
\end{array}\right) \int_{X} c_{1}(L)^{n} .
$$

Proof. Corollary 3.5 .2 follows from Theorem 3.5.1 by integration of 1 over $X$. A direct proof can be given though. The modulus of $p$ is a Morse function on $C_{\sigma} \backslash\left(\right.$ Base $\left.(p) \cup F_{0} \cup F_{\infty}\right)$, where $F_{0}$ (resp. $F_{\infty}$ ) is the fibre of 0 (resp. of $\infty$ ) of $p: X \rightarrow \mathbb{C} P^{1}$. Moreover, the index of every critical point of $|p|$ is $n-k$. As in the proofs of Propositions 1 and 2 in [15], we deduce that $\mathbb{E}\left(\mid\right.$ Crit $\left.p_{\left|C_{\sigma}\right|} \mid\right)$ is equivalent to $\left|\chi\left(C_{\sigma}\right)\right|$ as $d$ grows to infinity. Now,

$$
\chi\left(C_{\sigma}\right)=\int_{C_{\sigma}} c_{n-k}\left(C_{\sigma}\right)=\int_{X} c_{n-k}\left(C_{\sigma}\right) \wedge c_{k}\left(E \otimes L^{d}\right),
$$

while from the adjunction formula, $c\left(C_{\sigma}\right) \wedge c\left(E \otimes L^{d}\right)_{\mid C_{\sigma}}=c(X)$. Moreover, for $0 \leqslant i \leqslant k, c_{i}\left(E \otimes L^{d}\right)=\left(\begin{array}{c}k \\ i\end{array}\right) d^{i} c_{1}(L)^{i}+o\left(d^{i}\right)$, so that

$$
c\left(E \otimes L^{d}\right)=\left(1+d c_{1}(L)\right)^{k}+o\left(\left(1+d c_{1}(L)\right)^{k}\right) .
$$

From the formula $(1+x)^{-k}=\sum_{j=0}^{\infty}(-1)^{j} \frac{(k-1+j) !}{j !(k-1) !} x^{j}$, we then deduce that $c_{n-k}\left(C_{\sigma}\right)=$ $(-1)^{n-k}\left(\begin{array}{l}n-1 \\ k-1\end{array}\right) d^{n-k} c_{1}(L)^{n-k}+o\left(d^{n-k}\right)$ and finally that

$$
\chi\left(C_{\sigma}\right)=(-1)^{n-k}\left(\begin{array}{l}
n-1 \\
k-1
\end{array}\right) d^{n} \int_{X} c_{1}(L)^{n}+o\left(d^{n}\right) .
$$

Hence the result.

\section{References}

[1] A. Auffinger, G. Ben Arous, and J. Černý. Random matrices and complexity of spin glasses. Commun. Pure Appl. Math., 66(2):165-201, 2013.

[2] P. Bleher, B. Shiffman, and S. Zelditch. Universality and scaling of correlations between zeros on complex manifolds. Invent. Math., 142(2):351-395, 2000.

[3] E. Bogomolny, O. Bohigas, and P. Leboeuf. Quantum chaotic dynamics and random polynomials. J. Statist. Phys., 85(5-6):639-679, 1996.

[4] P. Bürgisser. Average Euler characteristic of random real algebraic varieties. $C$. R. Math. Acad. Sci. Paris, 345(9):507-512, 2007.

[5] J.-P. Dedieu and G. Malajovich. On the number of minima of a random polynomial. J. Complexity, 24(2):89-108, 2008.

[6] J.-P. Demailly. Estimations $L^{2}$ pour l'opérateur $\bar{\partial}$ d'un fibré vectoriel holomorphe semi-positif au-dessus d'une variété kählérienne complète. Ann. Sci. École Norm. Sup. (4), 15(3):457-511, 1982. 
[7] S. K. Donaldson. Symplectic submanifolds and almost-complex geometry. J. Differential Geom., 44(4):666-705, 1996.

[8] M. R. Douglas, B. Shiffman, and S. Zelditch. Critical points and supersymmetric vacua. I. Commun. Math. Phys., 252(1-3):325-358, 2004.

[9] M. R. Douglas, B. Shiffman, and S. Zelditch. Critical points and supersymmetric vacua. II: Asymptotics and extremal metrics. J. Differ. Geom., 72(3):381-427, 2006.

[10] H. Federer. Geometric measure theory. Die Grundlehren der mathematischen Wissenschaften, Band 153. Springer-Verlag New York Inc., New York, 1969.

[11] Y. V. Fyodorov. Complexity of random energy landscapes, glass transition, and absolute value of the spectral determinant of random matrices. Phys. Rev. Lett., 92(24):240601, 4, 2004.

[12] D. Gayet. Hypersurfaces symplectiques réelles et pinceaux de Lefschetz réels. J. Symplectic Geom., 6(3):247-266, 2008.

[13] D. Gayet and J.-Y. Welschinger. Exponential rarefaction of real curves with many components. Publ. Math. Inst. Hautes Études Sci., (113):69-96, 2011.

[14] D. Gayet and J.-Y. Welschinger. Betti numbers of random real hypersurfaces and determinants of random symmetric matrices. To appear in J. Eur. Math. Soc., arXiv:1207.1579, 2012.

[15] D. Gayet and J.-Y. Welschinger. What is the total Betti number of a random real hypersurface? J. Reine Angew. Math., published online, 10.1515/crelle2012-0062, 2012.

[16] D. Gayet and J.-Y. Welschinger. Lower estimates for the expected betti numbers of random real hypersurfaces. To appear in J. London Math. Soc., arXiv:1303.3035, 2013.

[17] L. Hörmander. An introduction to complex analysis in several variables. D. Van Nostrand Co., Inc., Princeton, N.J.-Toronto, Ont.-London, 1966.

[18] M. Kac. On the average number of real roots of a random algebraic equation. Bull. Amer. Math. Soc., 49:314-320, 1943.

[19] E. Kostlan. On the distribution of roots of random polynomials. In From Topology to Computation: Proceedings of the Smalefest (Berkeley, CA, 1990), pages 419-431. Springer, New York, 1993.

[20] A. Lerario and E. Lundberg. Statistics on Hilbert's sixteenth problem. arXiv:212.3823, 2013.

[21] X. Ma and G. Marinescu. Holomorphic Morse inequalities and Bergman kernels, volume 254 of Progress in Mathematics. Birkhäuser Verlag, Basel, 2007.

[22] B. Macdonald. Density of complex critical points of a real random $\mathrm{SO}(m+1)$ polynomial. J. Stat. Phys., 141(3):517-531, 2010. 
[23] M. L. Mehta. Random matrices, volume 142 of Pure and Applied Mathematics (Amsterdam). Elsevier/Academic Press, Amsterdam, third edition, 2004.

[24] J. Nash. Real algebraic manifolds. Ann. of Math. (2), 56:405-421, 1952.

[25] F. Nazarov and M. Sodin. On the number of nodal domains of random spherical harmonics. Amer. J. Math., 131(5):1337-1357, 2009.

[26] L. I. Nicolaescu. Critical sets of random smooth functions on compact manifolds. To appear in Asian J. Math. , arXiv:1101.5990, 2011.

[27] S. S. Podkorytov. The mean value of the Euler characteristic of an algebraic hypersurface. Algebra i Analiz, 11(5):185-193, 1999.

[28] P. Sarnak and I. Wigman. Topologies of nodal sets of random band limited functions. arXiv:1312.7858, 2013.

[29] H. Seifert. Algebraische Approximation von Mannigfaltigkeiten. Math. Z., 41(1):1-17, 1936.

[30] B. Shiffman and S. Zelditch. Distribution of zeros of random and quantum chaotic sections of positive line bundles. Comm. Math. Phys., 200(3):661-683, 1999.

[31] B. Shiffman and S. Zelditch. Addendum: "Asymptotics of almost holomorphic sections of ample line bundles on symplectic manifolds" [J. Reine Angew. Math. 544 (2002), 181-222; MR1887895 (2002m:58043)]. Proc. Amer. Math. Soc., 131(1):291-302, 2003.

[32] B. Shiffman and S. Zelditch. Random polynomials of high degree and Levy concentration of measure. Asian J. Math., 7(4):627-646, 2003.

[33] B. Shiffman and S. Zelditch. Number variance of random zeros on complex manifolds. Geom. Funct. Anal., 18(4):1422-1475, 2008.

[34] M. Shub and S. Smale. Complexity of Bezout's theorem. II. Volumes and probabilities. In Computational algebraic geometry (Nice, 1992), volume 109 of Progr. Math., pages 267-285. Birkhäuser Boston, 1993.

[35] M. Sodin. Lectures on random nodal portraits. lecture notes for a mini-course given at the St. Petersburg Summer School in Probability and Statistical Physics (June, 2012), 2012.

[36] M. Sodin and B. Tsirelson. Random complex zeroes. I. Asymptotic normality. Israel J. Math., 144:125-149, 2004.

[37] G. Tian. On a set of polarized Kähler metrics on algebraic manifolds. J. Differential Geom., 32(1):99-130, 1990.

[38] S. Zelditch. Real and complex zeros of Riemannian random waves. In Spectral analysis in geometry and number theory, volume 484 of Contemp. Math., pages 321-342. Amer. Math. Soc., Providence, RI, 2009. 
DAMIEN GAYET

UJF-GRENOBLE 1/CNRS

INSTITUT FOURIER,

UMR 5582 ,

Grenoble F-38401, France

damien.gayet@ujf-grenoble.fr

JEAN-YVES WELSCHINGER

UNIVERSITÉ DE LYON

CNRS UMR 5208

UNIVERSITÉ LYON 1

Institut CAMILLE JoRdAN

43 blvd. du 11 novembre 1918

F-69622 Villeurbanne cedex, France

welschinger@math.univ-lyon1.fr 